\title{
Anisotropic Dark Energy: Dynamics of Background and Perturbations
}

\author{
Tomi Koivisto \\ T.Koivisto@thphys. uni-heidelberg.de \\ Institute for Theoretical Physics, University of Heidelberg, 69120 Heidelberg,Germany \\ David F. Mota \\ D.Mota@thphys.uni-heidelberg.de \\ Institute for Theoretical Physics, University of Heidelberg, 69120 Heidelberg,Germany
}

\begin{abstract}
We investigate cosmologies where the accelerated expansion of the Universe is driven by a field with an anisotropic equation of state. We model such scenarios within the Bianchi I framework, introducing two skewness parameters to quantify the deviation of pressure from isotropy. We study the dynamics of the background expansion in these models. A special case of anisotropic cosmological constant is analyzed in detail. The anisotropic expansion is then confronted with the redshift and angular distribution of the supernovae type Ia. In addition, we investigate the effects on the cosmic microwave background (CMB) anisotropies for which the main signature appears to be a quadrupole contribution. We find that the two skewness parameters can be very well constrained. Tightest bounds are imposed by the CMB quadrupole, but there are anisotropic models which avoid this bound completely. Within these bounds, the anisotropy can be beneficial as a potential explanation of various anomalous cosmological observations, especially in the CMB at the largest angles. We also consider the dynamics of linear perturbations in these models. The covariant approach is used to derive the general evolution equations for cosmological perturbations taking into account imperfect sources in an anisotropic background. The implications for the galaxy formation are then studied. These results might help to make contact between the observed anomalies in CMB and large scale structure and fundamental theories exhibiting Lorentz violation.
\end{abstract}




\section{Contents}

1 Introduction $\quad 3$

2 Anisotropic Equation of State $\quad 5$

2.1 Parametrization . . . . . . . . . . . . . . . . . . 6

2.2 The matter content . . . . . . . . . . . . . . . . . . . 8

2.3 Evolution equations . . . . . . . . . . . . . . . . . . . . 10

3 The Background as a Dynamical System $\quad 11$

3.1 Fixed Points in the Axisymmetric Case . . . . . . . . . . . . . . . . . . . 11

3.2 An anisotropic Cosmological Constant . . . . . . . . . . . . . . . 15

3.2.1 Case I: Constant Isotropic Pressure . . . . . . . . . . . . . . . 16

3.2.2 Case II: Constant Anisotropic Pressure. . . . . . . . . . . . . . . . . 18

4 Observational Bounds from an Anisotropic Background Expansion 20

4.1 CMB Anisotropies . . . . . . . . . . . . . . . . . . . 20

4.2 SNIa Luminosities . . . . . . . . . . . . . . . . . . . . . . . . . 23

5 Inhomogeneous Cosmology 25

5.1 Covariant Perturbations . . . . . . . . . . . . . . . . . . 25

5.1 .1 Definitions. . . . . . . . . . . . . . . 26

5.1 .2 Nonlinear Equations. . . . . . . . . . . . . . . . . . 27

5.1.3 Linearized Evolution of Density. . . . . . . . . . . . . . . 28

5.1.4 Linearized Evolution of Shear. . . . . . . . . . . . . . . . . . . 29

5.2 Structure Formation . . . . . . . . . . . . . . . . . . . . 30

5.2 .1 Scalar Equations. . . . . . . . . . . . . . . . . . . . 30

5.2 .2 Case I: Small Anisotropy. . . . . . . . . . . . . . . . . . . . . 31

5.2 .3 Case II: "Adiabatic" Anisotropy. . . . . . . . . . . . . . . . . . . . 32

5.2 .4 Case III: Smooth Anisotropy. . . . . . . . . . . . . . . . . . . . . . 34

6 Conclusions and Discussion $\quad 35$

7 Appendix: Pedestrian notation for Bianchi I $\quad 37$ 


\section{Introduction}

There is a remarkable amount of observational evidence that the large-scale structure of the Universe resembles nearly the simplest and most symmetric imaginable system. Hence, it is well approximated by the flat geometry version of the Friedmann-Lemaitre models which are both homogeneous and isotropic. The fluctuations in the cosmic microwave background $(\mathrm{CMB})$ are a crucial probe of these properties, and therefore the recent detections of some unexpected features in the CMB temperature anisotropies have raised a lot of speculations about the need to reconsider some of the basic cosmological assumptions. A hemispherical asymmetry has been reported [1]. The angular correlation spectrum seems to be lacking power at the largest scales [2]. The alignment of the quadrupole and octupole (the so called Axis of Evil [3]) could also be seem as an extra-ordinary and unlikely result of statistically isotropic perturbations, even without taking into account that these multipoles happen also to be aligned to some extent with the dipole and with the equinox. The Axis of Evil does not show any correlation with the lack of angular power [4]. Given the a posteriori nature of these considerations, the cosmic variance (and the inevitable arbitrariness in any statistics), the statistical significance of all these anomalies is indeed a debatable issue [5-8].

Various possible cosmological effects have been proposed as possible explanations for those anomalous features. For instance, to introduce a preferred axis, one has to generate some isotropy breaking. According to whether this occurs at an early time or at late times, one may classify the models into those in which an originally isotropic CMB fluctuation is distorted on its way to us [9] and into those whose statistical anisotropy is imprinted in the primordial fluctuations [10]. A primordial imprint has been suggested to be left by isotropization happening during inflation [11,12], parity violating couplings [13] vector field gradients [14] or non-standard spinors [15]. On the other hand, the apparent alignments could also be caused by local effects in our neighborhood. Notice, however, that the Axis of Evil has been argued to probably persist after foreground removal [16]. Local voids have been though suggested as its possible origin [17] (and quite interestingly such voids could explain the present days acceleration too [18]).

In the case $\mathrm{CMB}$ is distorted during the late acceleration, the signatures of anisotropy would automatically be seen at the smallest multipoles of the CMB, since the perturbation wavelengths corresponding to these angles enter inside the horizon at the same epoch that the dark energy dominance begins. Therefore one would have one coincidence problem less. The apparent statistical anisotropy has been associated with dark energy in the form of vector fields [19-22], shear viscous fluids [23] and elastic solids [24]. See also [25-28] for other investigations. In general, if dark energy is something else than the cosmological constant $\Lambda$-term, one expects it to have anisotropic stresses at least at the perturbative level. This is also a generic prediction of modified gravity theories [29-35]. Moreover, it could be used to distinguish among scalar field theories in which the possible nonminimal coupling occurs only at the perfect fluid matter sector [36-39]. Such perturbative shear stresses have in general been attempted to be constrained through parameters describing directly the difference of the metric variables [40] and the viscous properties of generalized 
fluids [41-43]. Unfortunately, the perturbative anisotropic stress could escape detection, when its influences are restricted to so large scales that they are only seen through the amplitude of the first few multipoles of the CMB. In spite of that, in many specific models the Jeans scale of the (effective) dark energy is small enough for dark energy to form smallwavelength perturbations which might be probed by e.g. weak lensing experiments [4447]. Moreover, a statistical anisotropy due to dark energy would leave specific signatures which are not described alone by the amplitude of the CMB angular power spectrum $\sim\left\langle a_{l m}, a_{l m}^{*}\right\rangle$, where $a_{l m}$ are the coefficients of the spherical expansion of the anisotropy. Instead, correlations between different multipoles $l$ are predicted, and also $m$-dependent patterns (whereas statistical isotropy would predict, on average, the same value regardless of $m$ ). The theoretical prediction from statistical anisotropy to the CMB is thus the nondiagonality of the matrix $\left\langle a_{l m}, a_{l m}^{*}\right\rangle$.

Here we continue a previous study which investigated the implications and origins of an anisotropic acceleration [48]. There we studied exact anisotropic but homogeneous solutions of the cosmological equations. We now extend such investigation and generalise to the case where inhomogeneities are present and introduce a covariant framework for the study. The Bianchi I model is sufficiently simple to allow semi-analytical calculations, and it captures the basic effect present also in more complicated anisotropic models by featuring their important common property, direction-dependent expansion rates. Such metric is useful to describe magnetic fields [49] or their Yang-Mills generalizations [50]. As is well known, the anisotropy due to such fields tends to decay. Usually the constraints inferred on the Bianchi type I models have indeed been for cases in which the universe may initially be anisotropic but will then isotropize. Solutions which do not isotropize would require anisotropic matter sources, which in addition should dilute slower than radiation or dust. There has been no evidence that such cosmological sources would exist. However, as the universe is presently believed to be dominated by some source with unexplained negative pressure, a question we would like to ask is whether this pressure could also be anisotropic. This is of interest even without the apparent hints of statistical isotropy at the CMB.

After the study of Barrow on constraining anisotropic stresses in the late universe [49], we note that very recently there has been also interest in employing Bianchi I to study the CMB. Campanelli et al [51] describe magnetic fields present at the last scattering epoch resulting in an ellipsoidal universe. Similar effects have been proposed due to moving dark energy [52]. Rodrigues has suggested that an anisotropic cosmological constant [53] could result from an infrared noncommutative property of the spacetime. Longo has claimed that handedness of spiral galaxies is anisotropic [54] and that this, (together with the Axis of Evil) could be a result of large-scale magnetic fields [55]. If true, this would be a strong further motivation for our study since our model could unify magnetic and dark energy fields by describing Yang-Mills field or the mutually coupled system. In addition to these approaches, the anisotropic expansion rates have been exploited in inflationary considerations $[11,12,15,56,57]$. Then the appearance of anomalous features can be consistent, but does not necessitate, the existence of anisotropic sources. Inflation can smooth out initial anisotropies and inhomogeneities, but if the universe did not undergo 
too many e-folds of inflation $[58,59]$, some traces of such possible anisotropies could now be detectable at the largest scales. On the other hand, if one assumes isotropic initial conditions for the inflation, anisotropies could originate during inflation from a nonstandard energy source. This could be the same as dark energy.

We attack the problem at three fronts: phenomenologically, theoretically and observationally. In section 2 we present our phenomenological description of anisotropic sources in terms of the skewness parameters $\delta$ and $\gamma$, and derive the basic equations describing the model as a dynamical system. We then check the generic asymptotic evolution of the universe in some simple cases. This is a unifying description which can be used for any particular model of inflation or dark energy. Especially we contemplate the possibility of generalizing the usual $\Lambda$ term in such a way that it would correspond to a constant vacuum energy that may exert anisotropic pressure. Possibilities of unifying inflation and dark energy arise therefore naturally. Section 4 is devoted to the study of the implications of these models to cosmological observations. Explicit constraints are derived from the luminosity distance-redshift relationship of the supernovae of type Ia (SNIa) and the amplitude of the quadrupole anisotropy in the CMB. We find that the additional parameters we introduced, $\delta$ and $\gamma$, are well constrained. Finally, the last part of this article considers the inhomogeneities in the models considered here. In Section 5.1 we briefly introduce our notation for the covariant formalism and then present the general equations describing anisotropic cosmologies. Then we apply these general results into some interesting cases. In section 5.2 we study then large scale structure formation under various specific assumptions about the model. We conclude in section 6 .

\section{Anisotropic Equation of State}

We set up our framework within the Bianchi type I case. Usually the Bianchi models isotropize [60]. This is, in particular, true of the Bianchi I model with a perfect fluid content. Models that do not isotropize, have not been studied to such extent (though, for instance Ref. [61] presents exact solutions of inflationary Bianchi I cosmologies in the presence of various anisotropic sources). There has been good reasons for that: firstly, the modern cosmological observations, most importantly the satellite measurements of the CMB have established that the universe at large scales is isotropic to about one part in $10^{5}$. Secondly, one does not usually expect growing anisotropies, on the contrary to have them usually requires some imperfect and thus exotic matter sources. Thus it would seem that only a highly contrived and fine-tuned cosmology could exhibit the non-isotropizing property and consistency with the data. However, during the last decade, as mentioned in the introduction, evidence has accumulated against both of the presumptions above. Therefore, it is interesting to look for fixed points, especially scaling solutions, in the more general set-up allowing for direction-dependent expansion rates. It becomes then possible to see which kind of possibilities in general exist for anisotropic expansion histories, and whether they could occur naturally (i.e. without fine-tuning). We therefore perform a dynamical system analysis. Then, we will also consider the specific case of a generalized $\Lambda$ term. 


\subsection{Parametrization}

Consider a general fluid flow in a curved space-time. Assume the 4-velocity tangent to the fluid flow lines is $u^{a}$. This is the central object in the formulations below. Everything that follows will be defined in relation to this average flow. An useful tensor is then the projection tensor orthogonal to $u^{a}$, which is given by

$$
h_{a b}=g_{a b}+u_{a} u_{b} .
$$

The time derivative of any tensor $T^{a b \ldots}{ }_{c d \ldots}$ is understood as the derivative along the vector $u^{a}$, as

$$
\dot{T}^{a b \ldots}{ }_{c d \ldots} \equiv T^{a b \ldots}{ }_{c d \ldots ; e} u^{e} .
$$

Note that in general this differs from the derivative with respect to cosmic time, and agrees only for scalar fields. We also define ${ }^{(3)} \nabla$, the covariant derivative operator obtained by projecting the four-dimensional one as

$$
{ }^{(3)} \nabla_{e} T^{a b \ldots}{ }_{c d \ldots}=h_{a^{\prime}}^{a} h_{b^{\prime}}^{b} \ldots h_{c}^{c^{\prime}} h_{d}^{d^{\prime}} h_{e}{ }^{e^{\prime}} \ldots T^{a^{\prime} b^{\prime} \ldots}{ }_{c^{\prime} d^{\prime} \ldots ; e^{\prime}}
$$

The first covariant derivative of the $u^{a}$ is decomposed as follows:

$$
u_{a ; b}=\omega_{a b}+\sigma_{a b}+\frac{1}{3} \theta h_{a b}-a_{a} u_{b} .
$$

One calls $a_{a}=\dot{u}_{a}$ the acceleration, $\theta \equiv u_{; a}^{a}$ the expansion scalar, $\omega_{a b}=\omega_{[a b]}$ the vorticity tensor and $\sigma_{a b}=\sigma_{(a b)}$ the shear tensor. Both the shear tensor and vorticity tensor are traceless and orthogonal to $u^{a}$. It is useful to define a representative length scale $\ell$ by

$$
\frac{\dot{\ell}}{\ell}=\frac{1}{3} \theta
$$

This is a covariant generalization of the usual e-folding. Furthermore, the stress-energy tensor of a general fluid in curved spacetime is decomposed as follows:

$$
T_{a b}=\rho u_{a} u_{b}+p h_{a b}+2 q_{(a} u_{b)}+\pi_{a b},
$$

where $p$ is the pressure, $\rho$ is the energy density, $q_{a}$ is the energy flux and $\pi_{a b}$ the fluid viscosity.

In the following we consider the choice $u^{a}=\delta^{0}$, so that the fluid flow is given by the time-like unit vector. We also restrict ourselves to homogeneous cosmologies, and thus set any spatial gradient to zero. Furthermore, we assume that the vorticity vanishes. These assumptions, summarized as

$$
u^{a}=\delta_{0}^{a}, \quad{ }^{(3)} \nabla_{a} f=0, \quad \omega_{a b}=0
$$

characterize the Bianchi I cosmology. If we would further set $\sigma_{a b}=\pi_{a b}=0$, we would recover the Friedmann-Lemaître-Robertson-Walker (FLRW) universe.

In the Bianchi I spacetime, one needs three parameters to characterize the pressure of a fluid in all directions. In addition to the isotropic part which one gets as the trace part of Eq.(6),

$$
p=\frac{1}{3} h^{a b} T_{a b}
$$


two more parameters are needed to fully determine the degrees of freedom in the traceless and symmetric tensor

$$
p i_{a b}=\left(h_{(a}^{c} h_{b)}^{d}-\frac{1}{3} h^{c d}\right) T_{c d} .
$$

In Bianchi spacetime one may choose diagonal coordinate system, as we'll discuss later. It is occasionally claimed that one may consider the axisymmetric case without loss generality. It might then seem that one always choose the anisotropy axis parallel to one of the coordinate axes and thus, by considering a coordinate where anisotropy along one direction vanishes and due to the tracelessness requirement must in the remaining directions have an opposite sign and equal magnitude, describe the anisotropic pressure with full generality. However, rotation of the anisotropy axis would not preserve the diagonality of the system. The $3 x 3$ traceless symmetric tensor in (9) has 5 independent entries, but 3 of them may be fixed by choosing the spatial coordinate system. Thus the anisotropic pressure is characterized by two degrees of freedom. This resembles the anisotropic property of gravitational waves, which, at the level of linear perturbations are conventionally parametrized by the two modes of polarization with respect to the wavevector of perturbations. Since in the homogeneous background there is no obvious definition for such a wavevector, we instead use the following contractions for a fully covariant characterization of our anisotropic degrees of freedom:

$$
D \equiv \frac{1}{\rho^{2}} \pi_{a b} \pi^{a b}, \quad T=\frac{1}{\rho^{3}} \pi_{b}^{a} \pi_{a}^{c} \pi_{c}^{b} .
$$

Higher contractions would not be independent of these, but instead $\pi_{b}^{a} \pi_{d}^{c} \pi_{a}^{b} \pi_{c}^{d}=2 D^{2}$, $\pi_{b}^{a} \pi_{d}^{c} \pi_{a}^{e} \pi_{c}^{b} \pi_{e}^{d}=\frac{5}{6} T D$, etc. In the following we employ a combination of the quantities in (10) as

$$
\delta=-\frac{1}{3 \sqrt{2}} \frac{D+F}{\sqrt{F}}, \quad \gamma=\frac{\delta}{2} \pm \sqrt{D^{2}-\frac{3 \delta^{2}}{4}}
$$

where

$$
F^{3}=D^{3}-12 T^{2}+2 \sqrt{6} \sqrt{6 T^{4}-D^{3} T^{2}} .
$$

From these covariant expressions the use of this parametrization is far from obvious. Let us therefore make an excursion to the preferred coordinate system picked up by the symmetries of this model. In the diagonal system, where $\pi=\left(\pi_{x}, \pi_{y}, \pi_{z}\right)$, we have now

$$
\begin{aligned}
& \delta=-\frac{1}{3}\left(\pi_{x}-\pi_{y}\right) / \rho_{X}, \\
& \gamma=-\frac{1}{3}\left(2 \pi_{x}+\pi_{y}\right) / \rho_{X} .
\end{aligned}
$$

The use of these variables is mainly motivated by their straightforward interpretation as generalized equations of state (see the Appendix) and concordance with previous notations. The parameters $\delta$ and $\gamma$ may be interpreted simply as differences of the pressure along the $x$ and the pressure along the $y$ and $z$ axes, respectively. The axisymmetric cases are now seen to correspond to the three possible cases that either $\delta=0, \gamma=0$, or $\delta=\gamma$. One might get rid of the ambiguity by relabeling the axes in such a way if the pressure along 
any two axes is equal, the third one is called $x$ (a general rotation is not of course allowed by the symmetries). In the similar way, we will parametrize the shear stress of the metric as:

$$
\begin{aligned}
R & =\frac{3}{\theta}\left(\sigma_{x}-\sigma_{y}\right), \\
S & =\frac{3}{\theta}\left(2 \sigma_{x}+\sigma_{y}\right) .
\end{aligned}
$$

Again, this is just a change of variables which can be convenient for some purposes. The dimensionless variables $R$ and $S$ might be interpreted as the fractional difference of expansion rates between the $y$ and $x$ (for $R$ ) and between the $z$ and $x$ (for $S$ ). The notations (13) and (14) have been introduced in Ref. [49]. Again, the straightforward interpretation of the shears in the metric sector, $R$ and $S$, is confined to the preferred coordinate system, but these quantities can be defined fully covariantly in a way completely analogous to coefficients of the shear in matter sector, $\delta$ and $\gamma$ in Eq.(10).

\subsection{The matter content}

As the contents of the Universe we consider a two-fluid system, where only one of the fluids is responsible for possible nonstandard (anisotropic) properties. We have then a perfect fluid with an energy-momentum tensor (we denote it with index $m$ ) and an imperfect energy-momentum tensor (this we denote with index $X$ )

$$
T_{a b}=\rho_{m}\left(u_{a} u_{b}+w_{m} h_{a b}\right)+\rho_{X}\left(u_{a} u_{b}+w_{X} h_{a b}\right)+\pi_{a b},
$$

where $w_{m}$ is the equation of state parameter, $w_{m}=1 / 3$ for radiation and $w_{m}=0$ for dust (and something in between for their mixture). We also consider an imperfect fluid, which we allow to have the most general energy-momentum tensor compatible with the assumptions (7). The shear stress $\pi_{a b}$ is due to this component solely, and therefore we drop the $X$ from this quantity.

The continuity equations are then given by the divergence of the energy-momentum tensors. We let the two components also interact, and thus allow nonzero divergence for the individual components.

$$
\begin{aligned}
& \dot{\rho}_{m}+\left(1+w_{m}\right) \theta \rho_{m}=Q \rho_{m}, \\
& \dot{\rho}_{X}+\left(1+w_{X}\right) \theta \rho_{X}+\sigma_{a b} \pi^{a b}=-Q \rho_{m} .
\end{aligned}
$$

where $Q$ describes the coupling. If there are no interactions, one finds that the matter density scales as $\rho_{m} \sim\left(\ell^{3}\right)^{-1-w_{m}}$. For most of the study, we will simply neglect the coupling between the $X$ and the isotropic component. However, we keep it for the sake of generality, since we will later be interested in finding cosmological scaling solutions.

The system is of course not closed until we have determined the properties of the imperfect stresses $\pi_{\mu \nu}$. This is equivalent to specifying the anisotropy parameters $\delta$ and $\gamma$ in Eq.(13). In the case of viscous fluids [62], these stresses are related to the expansion factors. A covariant form for the viscosity generated in the fluid flow is

$$
\pi_{a b}=\varsigma\left(u_{a ; c} h_{b}^{c}+u_{b ; c} h_{a}^{c}-\frac{2}{3} u_{; c}^{c} h_{a b}\right)+\zeta u_{; c}^{c} h_{a b} .
$$




\begin{tabular}{|c|c|c|c|}
\hline Model & $\delta$ & $\gamma$ & $w_{X}$ \\
\hline Isotropic fluid & 0 & 0 & $w_{X}$ \\
\hline String & 0 & 1 & $1 / 3$ \\
\hline Domain wall & 1 & 1 & $2 / 3$ \\
\hline Magnetic field & 0 & -2 & $1 / 3$ \\
\hline Viscous fluid & $\frac{2 \varsigma R \theta}{9 \rho}$ & $\frac{2 \varsigma S \theta}{9 \rho}$ & $w_{X}-\zeta \frac{\theta}{\rho}$ \\
\hline$\Lambda$, case I & $\frac{h}{3} \theta(2 S-R)$ & $\frac{h}{3} \theta(S-2 R)$ & -1 \\
\hline$\Lambda$, case II & $\delta($ free $)$ & $\gamma($ free $)$ & $-1-\frac{1}{3}(3-2 R+S) \delta-\frac{1}{3}(3+R-2 S) \gamma$ \\
\hline
\end{tabular}

Table 1. Models with anisotropic equations of state.

Now the conservation equations $T_{; a}^{a b}=0$ reduce to the Navier-Stokes equations in the nonrelativistic limit. Here $\varsigma$ is the shear viscosity coefficient, and $\zeta$ represents bulk viscosity. Heuristically, they describe the resistance of fluid flow to external stresses. Such result in acceleration (derivatives of the velocity four-vector) which are then compensated by effect of $\pi_{a b}$. The bulk viscous part reacts to volume-changing stresses as it is proportional to the expansion scalar; in cosmology it gives an extra Hubble friction. The shear viscous part corresponds to the the symmetrized and traceless part of the acceleration, thus reacting to an anisotropic stress. Ultimately the coefficients $\zeta$ and $\varsigma$ should be determined from the microscopic kinetic theory. Calculating the tensor (18) explicitly with the help of the covariant definitions of section (5.1), one can write the equations of state as

$$
w=w_{X}-\left[3 \zeta+\frac{2}{3 \rho}(R+S) \varsigma\right] \frac{\theta}{3 \rho}, \quad \delta=\frac{2 \varsigma R \theta}{9 \rho}, \quad \gamma=\frac{2 \varsigma S \theta}{9 \rho} .
$$

One notes even with nonzero shear viscous coefficient $\varsigma$, one does not generate shear in the metric. In fact, with a positive coefficient $\varsigma$ (which is required by second law of thermodynamics), the viscous property of the fluid in fact tends to decrease the amount shearł. A bulk viscosity [63-66] could also have interesting consequences in this framework; however we do not focus further on the Navier-Stokes type of stress in the present study.

There are also several examples of imperfect cosmological sources with constant equations of state. A magnetic field along the $z$-direction [67] could be modeled by $w_{X}=1 / 3, \delta=0, \gamma=-2$. A string with a constant tension along the $z$-direction [68] could be described by $w_{X}=1 / 3, \delta=0, \gamma=1$. An anisotropic cosmological constant is described by $w_{X}=-1$. We mention these possibilities in Table 1 . We will return to these cases later. Note that our description with constant skewness parameters unifies and generalizes all of the mentioned models. Vector fields will be considered in much more detail in elsewhere [22]; we have found that such models can also feature constant equations of state $\delta$ and $\gamma$ in special cases, though they in general are dynamical, depending on the potentials and couplings [22]. We have gathered some other examples of imperfect matter in Table 1.

$\ddagger$ This may be seen by plugging the $\delta$ and $\gamma$ in the evolution equations $(25,26)$. 


\subsection{Evolution equations}

The generalized Friedmann equation can be written as

$$
\theta^{2}=3\left(\rho_{m}+\rho_{X}+\sigma^{2}\right) .
$$

We also define the dimensionless density fractions

$$
\Omega_{m} \equiv 24 \pi G \frac{\rho_{m}}{\theta^{2}}, \quad U \equiv \frac{\rho_{X}}{\rho_{m}+\rho_{X}} .
$$

We introduce $\Omega_{m}$ to quantify the amount of matter in analogy to the standard cosmology as the percentage of matter contribution to the square of the average expansion rate. Note that there is an ambiguity when referring to matter densities since, the generalized Friedmann Eq. (20) implying

$$
\Omega_{m}=(1-U)\left[1-3 \sigma^{2} / \theta^{2}\right],
$$

$\Omega_{m}$ is different from the matter density divided by the total density (which is $1-U$ ).

Our dynamical variables are then the density fraction $U$, and the two shear anisotropies $R$ and $S$. As the time variable we define $x=\log \ell$. In the following a prime denotes derivative with respect to that. The evolution equations for these are then

$$
\begin{aligned}
& U^{\prime}=U(U-1)\left[\pi^{a b} \sigma_{a b}+3\left(w_{X}-w_{m}\right)\right]-U Q, \\
& R^{\prime}=-\left(3+\frac{\theta^{\prime}}{\theta}\right) R+9 \delta\left(\frac{3 \sigma^{2}}{\theta^{2}}-1\right) U, \\
& S^{\prime}=-\left(3+\frac{\theta^{\prime}}{\theta}\right) S+9 \gamma\left(\frac{3 \sigma^{2}}{\theta^{2}}-1\right) U .
\end{aligned}
$$

One notes that only with non-vanishing skewness parameters, shear could be generated also in an initially isotropic situation. Also, if $\delta=\gamma=0$, even initially nonzero $R$ or $S$ would typically decay. Although the coupling term $Q$ does not complicate this system much, but appears only in the evolution equation for $U$, its presence of course can change the dynamics completely. As mentioned earlier, we consider mainly $Q=0$ case in this paper, but keep the coupling in the equations for the sake of generality. The two last equations can be written explicitly in terms of solely $R, S$, and $U$ as

$$
\begin{aligned}
& S^{\prime}=\frac{3}{2}\left(1-\frac{3 \sigma^{2}}{\theta^{2}}\right)\left\{S\left[U\left(w_{X}-w_{m}\right)+w_{m}-1\right]-6 \gamma U\right\} \\
& R^{\prime}=\frac{3}{2}\left(1-\frac{3 \sigma^{2}}{\theta^{2}}\right)\left\{R\left[U\left(w_{X}-w_{m}\right)+w_{m}-1\right]-6 \delta U\right\}
\end{aligned}
$$

In the following we will refer to an effective equation of state, which we define as

$$
w_{e f f} \equiv-\frac{2}{3} \frac{\theta^{\prime}}{\theta}-1,
$$

if $\sigma^{2}=0$, the universe expands as if dominated by a fluid with $w_{m}=w_{e f f}$; if $\sigma^{2} \neq 0$, the average of the three effective equations of state in each direction is $w_{\text {eff }}$. 


\section{The Background as a Dynamical System}

\subsection{Fixed Points in the Axisymmetric Case}

In the Friedmann-Lemaitre universe it has been proven difficult to address the coincidence problem by finding a model entering from a matter-dominated scaling solution to an accelerating scaling solution. Allowing the presence of three expansion rates perhaps opens up the possibility of finding more such scaling solutions which might eventually help to understand the coincidence problem. It is also possible to model inflation within this context (an interesting case then describes inflation as an era of primordial isotropization to the solution $R=S=0$ from a more general initial state). Therefore we consider the asymptotic behaviours of the universe. In the simplest anisotropic case we may assume: 1) axisymmetry, $S=\gamma=0,2)$ constant equations of state and skewness parameter $\dot{w}_{m}=\dot{w}=\dot{\delta}=0$, and to begin with, also 3) no coupling between the components $Q=0$. To end the analysis, we briefly review the consequences of relaxing the assumption 3 ). The results are summarized in the Table (2), and also discussed in some detail below. Please note that now $R$ can be considered as a shorthand notation for the expansion-normalized invariant shear

$$
R=3 \sqrt{3} \frac{\sigma}{\theta}
$$

and similarly $\delta$ corresponds to the density-normalized invariant shear

$$
\delta=\frac{\pi}{\sqrt{3} \rho} .
$$

Our system has 5 fixed points. With the assumptions 1)-3), there are two fixed points corresponding to matter domination. One is the

- FLRW solution

$$
R=0, \quad U=0 .
$$

We assume $w_{m}<1$. Then the FLRW solution is a stable node when $w+\delta>w_{m}$, otherwise a saddle point $§$.

- An anisotropically expanding empty universe

$$
R= \pm 3, \quad U=0
$$

is also a fixed point. This is a saddle point in the $R=+3$ case when $\delta<w-w_{m}$, and in the $R=-3$ case when $\delta>\left(w_{m}-w\right) / 3$ and otherwise unstable. This is the so called Kasner solution, where the metric may be described by

$$
d s_{\text {Kasner }}^{2}=-d t^{2}+t^{2 K_{a}} d x^{2}+t^{2 K_{b}} d y^{2}+t^{2 K_{c}} d z^{2},
$$

where the Kasner exponents satisfy

$$
1=K_{a}+K_{b}+K_{c}=K_{a}^{2}+K_{b}^{2}+K_{c}^{2} .
$$

$\S$ In this section $w$ corresponds to the equation of state in the $x$ - and $y$ directions. Thus $w_{X}=w+\delta$, if $w_{X}$ stands for the average of the principal pressures. 
In the present case, due to the axisymmetry, the exponents $K_{a}=K_{c}$ are of course equal, but we wrote the general form for later convenience. This solution is relevant near the singularity, $t \rightarrow 0$, where the matter may be neglected, $\rho_{m} / H^{2} \sim \rho / H^{2} \approx 0$. This solution may be realized in two ways $\|$. Firstly, one may have expansion in two and contraction in one direction, so that $K_{a}=-1 / 3$ and $K_{b}=K_{c}=2 / 3$ (or let the two directions change roles $a \leftrightarrow b$ to get the solution with $R=-3$ ). Secondly, one may have two static directions and one expanding direction, corresponding to $K_{a}=-1 / 3$ and $K_{b}=K_{c}=0$ (or again let the two directions change roles $a \leftrightarrow b$ to get the solution with $R=-3$ ).

- An anisotropically expanding universe dominated by dark energy,

$$
R= \pm 3, \quad U=1
$$

is also a fixed point of our system. The pancake solution with $R=+3$ is a saddle point if $w>1+\delta$ or $w<w_{m}+\delta$, and the cigar solution with $R=-3$ is a saddle point if $w+3 \delta>1$ or $w+3 \delta<w_{m}$. Otherwise they are unstable. On the average, the equation of state is for a stiff fluid, $w_{\text {eff }}=1$.

- The anisotropic fixed point

$$
R=\frac{6 \delta}{w+\delta-1}, \quad U=1
$$

represents a balanced expansion in different directions. Assuming here for simplicity that $w_{m}=0$ and restricting to the case $w<w_{m}$, this can be a stable node if either $\delta+w>1$ or

$$
1+6 \delta+\sqrt{1+16 w(w-1)}>2 w \wedge 2 w+\sqrt{1+16 w(w-1)}>1+6 \delta .
$$

We have a saddle point if either

$$
3 \delta+w<1 \wedge 2 w+\sqrt{1+16 w(w-1)} \leq 1+6 \delta
$$

or

$$
1+\delta>w \wedge 1+6 \delta+\sqrt{1+16 w(w-1)} \leq 2 w
$$

- The scaling solution

$$
R=\frac{3\left(w+\delta-w_{m}\right)}{2 \delta}, \quad U=\frac{\left(w+\delta-w_{m}\right)\left(w_{m}-1\right)}{3 \delta^{2}-2 \delta\left(w-w_{m}\right)-\left(w-w_{m}\right)^{2}} .
$$

is finally the fifth fixed point of our system. Considering again only cases when $w<w_{m}=0$, this is a saddle point if either

$$
\delta>0 \wedge 2 w+\sqrt{1+16 w(w-1)} \geq 1+6 \delta
$$

or

$$
\delta<0 \wedge 1+6 \delta+\sqrt{1+16 w(w-1)} \geq 2 w .
$$

General stability conditions for this point are more complicated. Instead of trying to find feasible analytic expressions for them, we resort to an empirical study.

$\|$ In terms of the expansion rates introduced in the appendix, the mentioned two possibilities correspond to $\dot{a} / a=-1 /(3 t), \quad$ and $\quad \dot{b} / b=\dot{c} / c=2 /(3 t)$, and $\dot{a} / a=1 / t, \quad$ and $\quad \dot{b} / b=\dot{c} / c=0$. 
To study when the solutions are relevant for the dark energy problem, we use a dustdominated FLRW universe, $w_{m}=R=S=0$, as the initial condition. Then the outcome is uniquely determined by dark energy properties, $w$ and $\delta$. The numerical results, shown in Figs. $(1,2)$ and summarized in Fig. 3, agree completely with our analytical considerations.
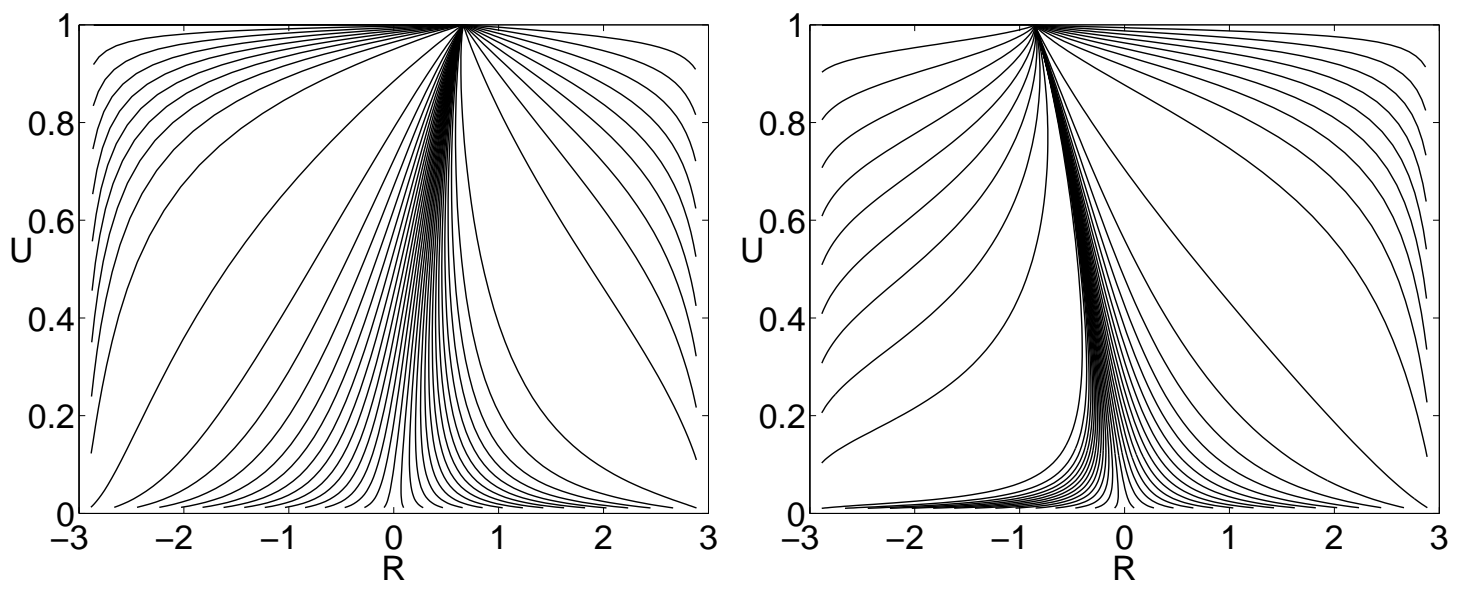

Figure 1. Phase portraits in the cases $\delta=-0.25$ (right) and $\delta=0.25$ (left) when $w=-1$. The solution (28) is at $R=U=0$, and the fixed points (29) and (32) are at the four corners of the portraits. In both cases the fixed point (33) attracts trajectories from everywhere.
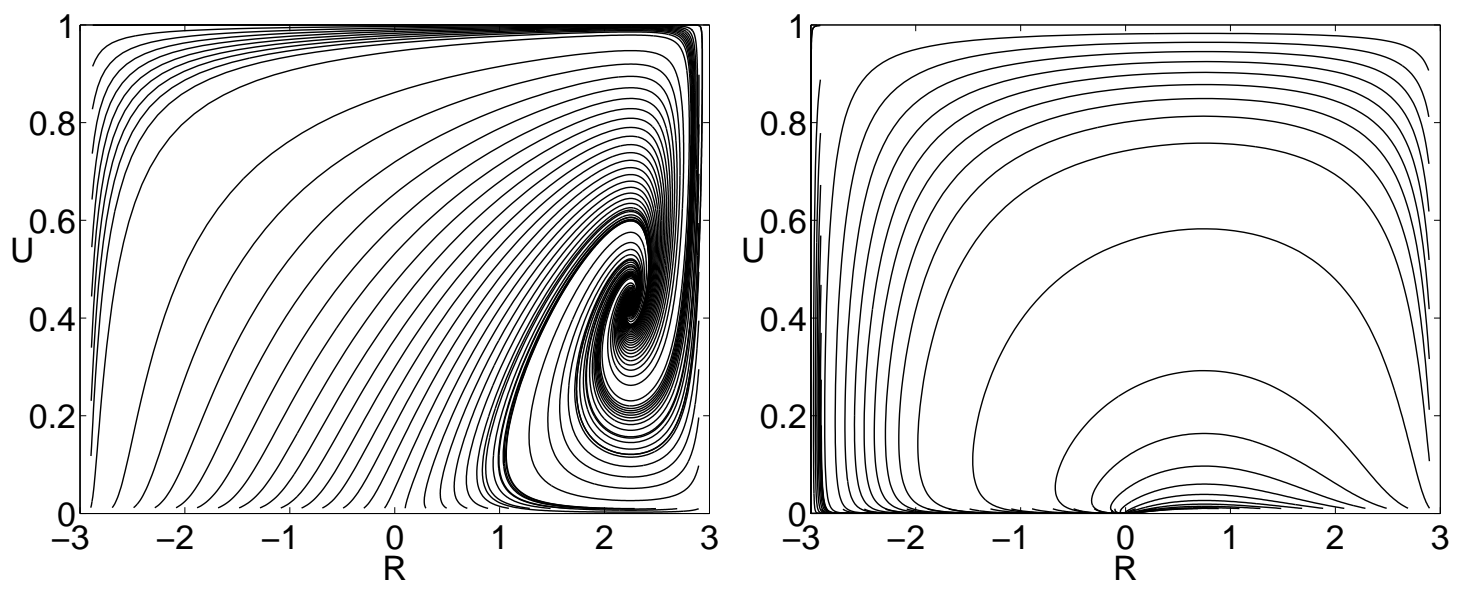

Figure 2. Phase portraits in the cases $\delta=-2$ (right) and $\delta=2$ (left) when $w=-1$. The solution (28) is at $R=U=0$, and the fixed points (29) and (32) are at the four corners of the portraits. In right panel all trajectories lead to the scaling solution all (34). The left panel shows isotropizations in the example with $w+\delta>w_{m}$.

Let us finally consider relaxing the assumption 3) to include a coupling $Q$. We assume it is constant. This may be motivated by the analogy with scalar fields (exponential interaction term in the Lagrangian results in constant $Q$ for scaling solutions), and can also be realized with vector fields having matter couplings, as we show in another publication 


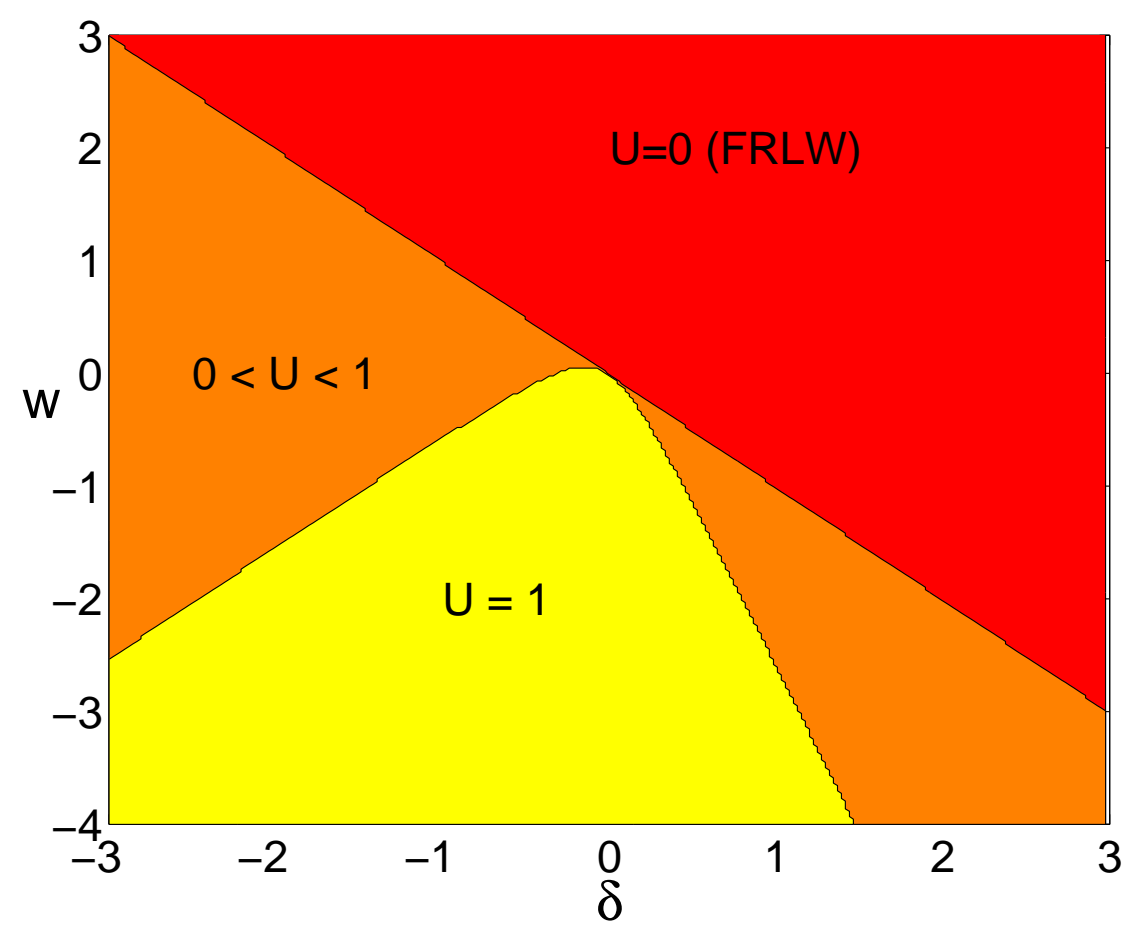

Figure 3. The asymptotic state of the universe starting from an Einstein-deSitter stage. The future fate depends on the dark energy properties $w$ and $\delta$ and is classified into three possibilities. If $\delta+w<0$, the isotropically expanding dust domination (solution (28) where $U=0$ ) continues forever. Otherwise, the universe will end up expanding anisotropically and either dominated by dark energy (solution (33) where $U=1$ ) or exhibiting a scaling property (solution (34) where $0<U<1$ ). Fig. (1) showed phase portraits of two $U=1$ models, the right panel of Fig. (2) showed an example of the $0<U<1$ case, and finally the left panel of Fig. (2) showed isotropizations in the $U=0$ (here we refer to the asymptotic value of $U$ ).

with explicit examples [22]. Then the dark energy dominated anisotropic solution Eq.(32) would turn into an anisotropic scaling solution

$$
R= \pm 3, \quad U=1-\frac{Q}{3\left(w_{m}-w\right)+(3 \mp 6) \delta},
$$

and where the amount of matter is proportional to the strength of the coupling $Q$.

The scaling solution (34) generalizes in the presence of a coupling to

$$
\begin{aligned}
& R=\frac{15 \delta^{2}+(-3+Q+3 w)\left(w-w_{m}\right)+\delta\left(Q+6 w-3\left(1+w_{m}\right)\right) \pm \sqrt{r}}{4 \delta(-1+\delta+w)} \\
& U=\frac{9 d^{2}-\delta\left(3+Q+6 w-9 w_{m}\right)-\left(3+Q+3 w-6 w_{m}\right)\left(w-w_{m}\right) \pm \sqrt{r}}{6\left(3 \delta+w-w_{m}\right)\left(\delta-w+w_{m}\right)}
\end{aligned}
$$

where

$$
\begin{aligned}
& r=\left(-9 \delta^{2}+\delta\left(3+Q+6 w-9 w_{m}\right)+\left(3+Q+3 w-6 w_{m}\right)\left(w-w_{m}\right)\right)^{2}- \\
& 12\left(3 \delta+Q+3 w-3 w_{m}\right)\left(3 \delta+w-w_{m}\right)\left(w_{m}-1\right)\left(\delta-w+w_{m}\right) .
\end{aligned}
$$

Thus the coupling modifies both the relative amount of anisotropy and the relative amount 


\begin{tabular}{|c|c|c|c|c|c|}
\hline Solution & Eqs & $w_{\text {eff }}$ & $R$ & $U$ & Coupling \\
\hline FLRW & $(28)$ & $w_{m}$ & 0 & 0 & - \\
\hline Empty & $(29)$ & 1 & \pm 3 & 0 & - \\
\hline DE 1 & $(32)$ & $1 \mp 2 \delta$ & \pm 3 & 1 & See Eq.(35) \\
\hline DE 2 & $(33)$ & $1-\frac{4 \delta^{2}}{\delta+w-1}$ & $\frac{6 \delta}{w+\delta-1}$ & 1 & - \\
\hline Scaling & $(34)$ & $w_{m}-\frac{4 \delta^{2}\left(w_{m}-1\right)}{\left(3 \delta+w-w_{m}\right)\left(\delta-w+w_{m}\right)}$ & $\frac{3\left(w+\delta-w_{m}\right)}{2 \delta}$ & $\frac{\left(w+\delta-w_{m}\right)\left(w_{m}-1\right)}{3 \delta^{2}-2 \delta\left(w-w_{m}\right)-\left(w-w_{m}\right)^{2}}$ & See Eq.(36) \\
\hline
\end{tabular}

Table 2. Fixed points in the axisymmetric case, with constant equations of state $w$, $w_{m}$ and $\delta$. The effective equation of state $w_{\text {eff }}$ is defined in Eq.(27). The last column indicates whether the solution can be modified by a coupling. The fixed point corresponds to an anisotropic expansion unless $R=0$.

of matter. An isotropic scaling solution is now possible with a nonzero coupling and $\delta=0$. We list a summary of the fixed points found in this subsection in the Table (2).

The rest of the fixed points, Eqs.(28), (29) and (34) would retain their form in the presence of a coupling term.

\subsection{An anisotropic Cosmological Constant}

We will show that there does not exist an anisotropic generalization of the cosmological constant with constant equations of state. Then we consider two different classes of timedependent cosmological terms with constant energy density.

The continuity equation (93) for a component with general $w, \delta$ and $\gamma$ reads

$$
\begin{array}{ll}
\dot{\rho}_{\Lambda}+\theta\left(1+w_{\Lambda}\right) \rho_{\Lambda}+\pi_{a b} \sigma^{a b} & =0 \\
\dot{\rho}_{\Lambda}+\theta\left[\left(1+w_{\Lambda}\right)+\frac{1}{3} \gamma(R-2 S)+\frac{1}{3} \delta(-2 R+S)\right] \rho_{\Lambda}=0 .
\end{array}
$$

We have added the subscript to emphasize we are discussing a cosmological constant term. In the second line we wrote open the component contributions to the invariant shear. As we then want $\dot{\rho}_{\Lambda}=0$, the square brackets term should vanish identically. If we have time-independent equations of state, we cannot consistently model an anisotropic $\Lambda$ term. Obviously, the constant terms in the square brackets should then vanish, and we should set $w_{X}=-1$. Since there also appears $R$ and $S$, which in general depend on time, the linear combination

$$
X \equiv \frac{3}{\theta} \pi_{a b} \sigma^{a b}=(R-2 S) \gamma+(-2 R+S) \delta
$$

should however be zero. To see how this is impossible, one derives an evolution equation for $X$ :

$$
\begin{aligned}
\dot{X} & =-\left(\theta+\frac{\dot{\theta}}{\theta}\right) X+\frac{6 \pi^{2}}{\rho_{X} \theta} \\
& =-\left(\theta+\frac{\dot{\theta}}{\theta}\right) X+3[\gamma(\delta-2 \gamma)+\delta(\gamma-2 \delta)]\left(\frac{3 \sigma^{2}}{\theta^{2}}-1\right) \theta U .
\end{aligned}
$$


The second term will always enforce $X$ to evolve unless $\delta=\gamma=0$. Note that the second term does not vanish since Eqs. $(25,26)$ set the shears evolving.

As a summary, we note that in terms of our covariant notation, the two conditions mentioned above correspond to the two statements

- $w_{\Lambda}=1$, i.e. the isotropic part of the pressure should be equal to minus the energy density, $p_{\Lambda}=-\rho_{\Lambda}$.

- $X=0$, i.e. the anisotropic part of the pressure should be orthogonal to the shear expansion, $\pi^{a b} \sigma_{a b}=0$.

If both of these conditions are satisfied, with constant $\delta$ and $\lambda$, these must be zero. We will therefore consider dynamical equations of state in the following subsection 3.2.1. However, it turns out that to find interesting cosmology one may have to violate one of the conditions above too. We will consider this in the later subsection 3.2.2.

3.2.1. Case I: Constant Isotropic Pressure. One may satisfy both of the two requirements stated above with time-varying skewness parameters. We introduce a parameter $h$ and write

$$
\begin{aligned}
\delta & =\frac{h}{3} \theta(2 S-R), \\
\gamma & =\frac{h}{3} \theta(S-2 R) .
\end{aligned}
$$

Then the anisotropic stress becomes

$$
\pi_{b}^{a}=\operatorname{diag}[0,-S+R, S,-R] h H,
$$

which indeed is, by construction, orthogonal to the metric shear

$$
\sigma^{a}{ }_{b}=\operatorname{diag}[0, R+S,-2 R+S, R-2 S] H / 3 .
$$

Since now also $w_{X}=-1$, both the energy density and the isotropic part of the pressure are constant. This prescription seems to be equivalent to the model introduced by Rodrigues [53], who also shows it is possible to associate such properties of the cosmological term with an infrared non-commutative property of the spacetime. The anisotropy arises from the deformation of the Poisson structure between the canonical momenta in such a way that the dynamics remain invariant under rescalings of the scale factors [53]. The noncommutativity coefficients are then proportional to the parameter $h$ (which in our case has dimension of $1 / \theta)$.

This would be difficult to apply for description of anisotropies in the present universe. The reason can be seen from skewness parameters (43) and (44). Since they are proportional to the shear, the model does not support spontaneous generation of anisotropy. If $R$ and $S$ vanish in the early universe, they will stay zero even when the $\Lambda$ term becomes dominant. Observable deviation from $\Lambda \mathrm{CDM}$ could occur if the system turned out to be unstable: the $\Lambda$ term could in principle react to even perturbatively small anisotropies by amplifying them into direction-dependent background expansion. Let us briefly study this 
possibility. After algebraic manipulation one may write the evolution equations as follows

$$
\begin{aligned}
& U^{\prime}=-3 U(U-1)\left(1+w_{m}\right), \\
& R^{\prime}=\frac{3}{2}\left(1-\frac{3 \sigma^{2}}{\theta^{2}}\right)\left[-4 h \theta S U-R\left(1+U-2 h \theta U+(U-1) w_{m}\right)\right], \\
& S^{\prime}=\frac{3}{2}\left(1-\frac{3 \sigma^{2}}{\theta^{2}}\right)\left[4 h \theta R U-S\left(1+U+2 h \theta U+(U-1) w_{m}\right)\right],
\end{aligned}
$$

where the Hubble factor can be expressed as

$$
h \theta=\frac{\hat{h}}{\left[U\left(1-\frac{3 \sigma^{2}}{\theta^{2}}\right)\right]^{1 / 2}}
$$

with the rescaled constant $\hat{h}$ which is now dimensionless, defined as $\hat{h} \equiv h \sqrt{24 \pi G \rho_{\Lambda}}$. The equation for $U^{\prime}$ is of the same form as in $\Lambda$ CDM cosmology. The solution is thus

$$
U(x)=\frac{1}{1+\left(\frac{1}{U_{0}}-1\right) e^{-3\left(1+w_{m}\right) x}},
$$

where $U_{0}$ is the relative amount of vacuum density today. It is also obvious that $R=S=0$ is a solution with any value of $w$. In fact the system has several fixed points.

- The Kasner solutions

$$
R=\frac{S}{2} \pm \sqrt{9-\frac{3}{4} S^{2}} .
$$

These correspond to the metric (30). Since we are not restricted here to a specific axisymmetric case, the Kasner exponents have only to satisfy the relations (31). The solutions with different $-3<S<2$ correspond to these different exponents. Since for the Kasner solution matter is negligible, the value of $U$ is irrelevant (though formally should be set either to $U=0$ or $U=1$ ). One quickly notices that this class of solutions is unstable: the anisotropy decays and matter becomes eventually dominant.

- The $\Lambda$ dominated isotropic solution

$$
R=S=0, \quad U=1 .
$$

If we then consider small perturbations $U=1+u, R=0+r, S=0+s$ about the $\Lambda$ dominated solution, we find that

$$
u \sim x^{-3\left(1+w_{m}\right)}, \quad \text { while } \quad r, s \sim x^{-3 \pm i \sqrt{3} \hat{h}} .
$$

Hence, the anisotropy decays, proportional to the inverse volume element. If anisotropy is inserted as an initial condition, the non-zero skewness parameters (43) and (44) only cause an oscillation about the usual diluting behaviour.

- The matter dominated isotropic solution

$$
R=S=0, \quad U=0 .
$$

One may also check that for the matter dominated solution, the perturbations go as

$$
u \sim x^{3\left(1+w_{m}\right)}, \quad r, s \sim x^{-3\left(1+w_{m}\right) / 2} .
$$


The negativity of the first exponent only signals that the fractional matter density is diminishing. The two last ones tell us that during matter domination the anisotropy decays too, though slower than during the $\Lambda$ domination, and to first order without oscillations.

For a cosmological evolution the relevant solutions are then the two last ones with $U=0$ and the other with $U=1$, and both having $R=S=0$. These will come out according to Eq.(49). The anisotropy is negligible by definition when the $\Lambda$ term can be neglected. Shear is not generated during the evolution of the universe, since anisotropic perturbations about the usual solution decay. We have checked numerically that this behaviour is general and is not restricted to the asymptotic regimes of cosmological evolution.

3.2.2. Case II: Constant Anisotropic Pressure. Let us now take a different approach, and keep $\delta$ and $\gamma$ as free parameters describing the anisotropy of the $\Lambda$ term. The Eq.(38) should still reduce to the identity $\dot{\rho}_{\Lambda}=0$. There is a simple prescription which achieves this by forcing the isotropic pressure to compensate for the anisotropic part:

$$
w_{\Lambda}=-1-\frac{1}{3} X
$$

The equations of state associated to the $\Lambda$-term can now be also different from -1 . Also in such cases the vacuum energy density stays constant, but then the pressure becomes direction dependent, forcing eventually the universe to expand anisotropically when the cosmological term is significantly large. The evolution equations for the background variables then reduce to

$$
\begin{aligned}
& U^{\prime}=-3 U(U-1)\left(1+w_{m}\right), \\
& S^{\prime}=\frac{1}{6}\left(9-R^{2}+R S-S^{2}\right)\left\{S\left[-U\left(1+\frac{1}{3} X+w_{m}\right)+w_{m}-1\right]-6 \gamma U\right\} \\
& R^{\prime}=\frac{1}{6}\left(9-R^{2}+R S-S^{2}\right)\left\{R\left[-U\left(1+\frac{1}{3} X+w_{m}\right)+w_{m}-1\right]-6 \delta U\right\}
\end{aligned}
$$

The equation for $U^{\prime}$ in (54) is of the same form as in $\Lambda$ CDM cosmology, and is given by Eq.(49). We have not found an analytic solution for the shears $R$ and $S$.

For simplicity, let us consider the axisymmetric case that $S=\gamma=0$. We then find several fixed points.

- The FLRW,

$$
R=0, \quad U=0,
$$

corresponding now to Eq.(28). It is easy to see that this point is always unstable. The matter domination will inevitably be followed by the cosmological constant dominated era, which is anisotropic whenever $\delta \neq 0$.

- An anisotropic matter domination,

$$
R= \pm 3, \quad U=0 .
$$

For dust matter this is always an unstable point. 
- An anisotropic $\Lambda$ domination I,

$$
R= \pm 3, \quad U=1 .
$$

For dust matter, this is a saddle point.

- An anisotropic $\Lambda$ domination II,

$$
R=\frac{1}{2 \delta}\left(3 \pm \sqrt{9+36 \delta^{2}}\right), \quad U=1 .
$$

One may show that when $w_{m}=0$, this is always an attractor. More specifically, introducing small perturbations about this solution, and constructing the matrix for the derivatives of these perturbations from the system (54), the eigenvalues $q_{u}, q_{r}$ of the matrix then are

$$
q_{u}=-3, \quad q_{r}=-\frac{3}{2 \delta^{2}} \sqrt{1+4 \delta^{2}}\left( \pm 1+\sqrt{1+4 \delta^{2}}\right) .
$$

Since these are negative regardless of the value of $\delta$, we know that the perturbations tend to decay.

A scenario then appears naturally, featuring a transition from the usual isotropic fixed point (56) to the anisotropic vacuum dominated point (58). This point coincides with the solution (57) when $\delta$ is very large. One notices that the asymptotic value of the shear $R$ goes to $R \rightarrow \pm 3$ when $\delta \rightarrow \pm \infty$. We have numerically verified that the universe always evolves along this track when begun from an isotropic initial stage. This then provides the possibility to generalize the standard $\Lambda \mathrm{CDM}$ cosmology in such a way that the universe features anisotropies at late times when the $\Lambda$-term begins to dominate.

If the matter content is dust, the equation of state for the total fluid in this model is given by

$$
w_{T O T}=-\left(1+\frac{X}{3}\right) U
$$

and the total sound speed squared is

$$
c_{\text {sTOT }}^{2}=\frac{1}{9} X^{\prime}\left(\frac{1}{\frac{1}{U}-1}\right)=\frac{\delta\left(R^{2}-9\right) U[18 \delta U+R(3+(3-\delta(3+2 R)) U)]}{81(U-1)},(61)
$$

assuming that $\delta$ is constant. One notes that this could be positive or negative. It vanishes when $\delta$ does: the usual $\Lambda \mathrm{CDM}$ universe is "silent" in the sense that the CDM is pressureless, no perturbations propagate in the (isotropic) $\Lambda$ medium. The anisotropy quantity $\xi$ is now

$$
\xi=\frac{2 H R}{1-\frac{1}{U}-\frac{2}{3} \delta R}
$$

It was expected that this is proportional to the shear in the expansion rate, $H R$.

In the Figure 4 we show the asymptotic state of the universe as a function of the skewness parameter $\delta$. In this numerically produced plot we also consider some cases where $\gamma$ does not equal zero, (though for simplicity we restricted to an axisymmetric case $S=\gamma=0$ in the analytic considerations). We return later to briefly consider the observational implications of this anisotropic generalization of $\Lambda$. 


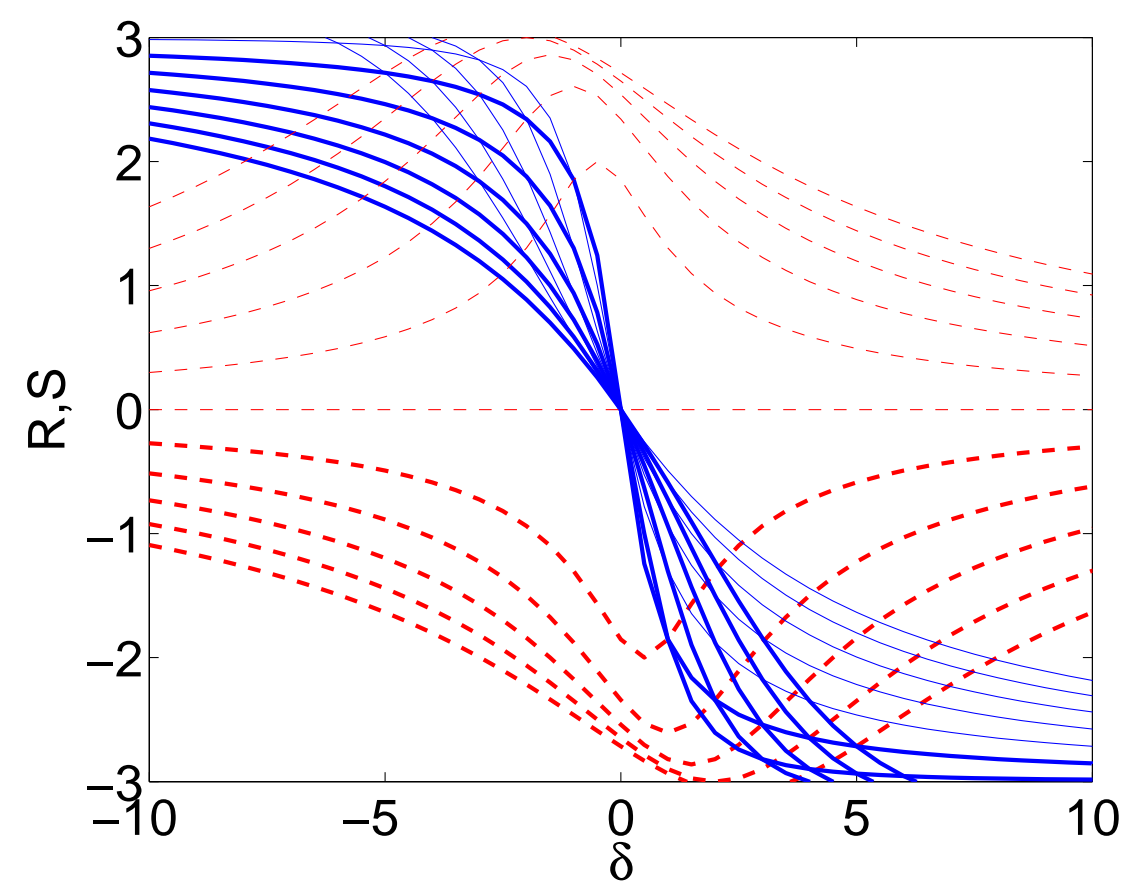

Figure 4. The asymptotic state of the universe starting from an Einstein-deSitter stage for the anisotropic generalization of cosmological constant having constant $\delta$ and $\gamma$ (case II, section 3.2.2). The $R$ (blue, solid lines) and $S$ (red, dashed lines) are given as function of $\delta$. The thick lines depict the cases $\gamma=1,2,3,4,5$, and the thin lines depict the case that $\gamma=0,-1,-2,-3,-4,-5$. The analytic solution (58) is recovered in the case $\gamma=0$. These curves show how this generalizes non-axisymmetric cases.

\section{Observational Bounds from an Anisotropic Background Expansion}

The part of this study focusing on homogeneous cosmology is ended by a comparison of the background dynamics of these models to the cosmological observations. In Section (2) we have made a phenomenological description of an anisotropic component in terms of the skewness parameters $\delta$ and $\gamma$, Eq.(13). The question of its observational consequences is taken under investigation now. We then focus on dark energy and an anisotropic background expansion. Observable effects which might have been imprinted during the early inflationary era, would appear to be more straightforwardly described as perturbations and thus not changing the FLRW predictions at zero order $[69,70]$. As we emphasise later, a proper treatment should take into account perturbations, in particular (the possibly anisotropic) initial conditions set at inflation [71]. However, as an attempt at a model-independent first constraint on the model, it is useful to estimate the bounds ensuing from the background expansion.

\section{1. $C M B$ Anisotropies}

A possible anisotropy will leave its imprint in the CMB. To calculate the CMB spectrum in a background with a Bianchi I metric Eq.(122), we begin from the geodesic equation for 
photons,

$$
\frac{d^{2} x^{\mu}}{d \lambda^{2}}+\Gamma_{\alpha \beta}^{\mu} \frac{d x^{\alpha}}{d \lambda} \frac{d x^{\alpha}}{d \lambda}
$$

where the only nonvanishing connection coefficients are (summations are suppressed for Latin indices in this section)

$$
\Gamma_{i i}^{0}=a_{i} \dot{a}_{i}, \quad \Gamma_{0 i}^{i}=\Gamma_{i 0}^{i}=\frac{\dot{a}_{i}}{a_{i}} .
$$

We are interested in the redshift of photons coming from different directions. The four vector $u^{\mu}=d x^{\mu} / d \lambda$ is subject to the null condition,

$$
u^{\mu} u_{\mu}=0=-\left(\frac{d t}{d \lambda}\right)^{2}+\sum_{i} a_{i}^{2}\left(P^{i}\right)^{2},
$$

where we call $u^{i} \equiv P^{i}$. Consider two photons, the first emitted at $t=t_{e}$ and the other a tiny bit $\tau$ later at $t=t_{e}+\tau$. The difference of the two null conditions gives

$$
u^{0} \frac{d \tau}{d \lambda}=\sum_{i} a_{i} \dot{a}_{i}\left(P^{i}\right)^{2} \tau(\lambda)+\mathcal{O}\left(\tau^{2}\right) .
$$

The definition of redshift is

$$
1+z\left(\lambda_{e}\right) \equiv \frac{\tau\left(\lambda_{r}\right)}{\tau\left(\lambda_{e}\right)},
$$

where $\tau\left(\lambda_{r}\right)$ is the time difference of the received signals. Differentiating Eq.(67) and using Eq.(66) yields

$$
\frac{d \log (1+z)}{d \lambda}=-\frac{1}{u^{0}} \sum_{i} a_{i} \dot{a}_{i}\left(P^{i}\right)^{2} .
$$

The $P^{i}$ can be obtained from the spatial components of the geodesic equation (63),

$$
\frac{d P^{i}}{d \lambda}+2 \frac{\dot{a}_{i}}{a_{i}} P^{i} u^{0}=0 .
$$

We find $P^{i} \sim a_{i}^{-2}$. Since one can always rescale the scale factors, let us fix $a_{i}\left(t_{0}\right)=1$ where $t_{0}$ indicates today. We then write our initial $P^{i}$ as $P^{i}\left(t_{0}\right)=\hat{p}_{i}$. Since one can reparameterize $\lambda$ without affecting the results, we choose now such a normalization that $\sum_{i} \hat{p}_{i}^{2}=1$. One may thus think about the unit vector $\hat{p}$ in terms of the angles $\left(\hat{p}_{x}, \hat{p}_{y}, \hat{p}_{z}\right)=(\sin \theta \cos \phi, \sin \theta \sin \phi, \cos \theta)$. Thus we have managed to parametrize the photon paths according to the angle they hit the observer today. Inserting this solution for $P^{i}$ and $u^{0}$ from Eq.(65) into the evolution equation for the redshift (68), one finds that it can be solved to yield

$$
1+z(\hat{\mathbf{p}})=\left(\sum_{i} \frac{\hat{p}_{i}^{2}}{a_{i}^{2}}\right)^{\frac{1}{2}} .
$$

Since the sum is equal to

$$
\sum_{i} \frac{\hat{p}_{i}^{2}}{a_{i}^{2}}=\frac{1}{a^{2}}\left[\sin ^{2} \theta\left(\cos ^{2} \phi+\frac{a^{2}}{b^{2}} \sin ^{2} \phi\right)+\frac{a^{2}}{c^{2}} \cos ^{2} \theta\right],
$$


it is easy to see that one can rewrite the result as

$$
1+z(\hat{\mathbf{p}})=\frac{1}{a} \sqrt{1+\hat{p}_{y}^{2} e_{y}^{2}+\hat{p}_{z}^{2} e_{z}^{2}}
$$

in terms of the eccentricities

$$
e_{y}^{2}=\left(\frac{a}{b}\right)^{2}-1, \quad e_{z}^{2}=\left(\frac{a}{c}\right)^{2}-1 .
$$

The temperature field is determined by this redshift by the relation

$$
T(\hat{\mathbf{p}})=\frac{T_{*}}{1+z(\hat{\mathbf{p}})},
$$

and the spatial average is

$$
4 \pi \bar{T}=\int d \Omega_{\hat{\mathbf{p}}} T(\hat{\mathbf{p}}) .
$$

The last scattering temperature $T_{*}$ does not depend on the direction, but the photons coming from different directions will be redshifted different amounts. The anisotropy field is then

$$
\frac{\delta T(\hat{\mathbf{p}})}{\bar{T}}=1-\frac{T(\hat{\mathbf{p}})}{\bar{T}} .
$$

The coefficients in the spherical expansion of this anisotropy field are called $a_{\ell m}$, and due to orthogonality of spherical harmonics $Y_{\ell m}$, are given by

$$
a_{\ell m}=\int d \Omega_{\mathbf{p}} \frac{\delta T(\hat{\mathbf{p}})}{\bar{T}} Y_{\ell m}^{*}
$$

The multipole spectrum can be described by

$$
Q_{\ell}=\sqrt{\frac{1}{2 \pi} \frac{\ell(\ell+1)}{(2 \ell+1)} \sum_{m=-\ell}^{\ell}\left|a_{\ell m}\right|^{2} .}
$$

One may expand the redshifts (72) in the eccentricities (73). Then one notes that the $a_{\ell m}$ will be real (since there is only even dependence on the polar angle in the anisotropy field and the imaginary parts of the $e^{i m \phi}$ integrate to zero), and that for all odd $\ell$ the $a_{\ell m}$ will vanish (since only even powers of the azimuthal appear in the expansion). To first order in $e_{x, y}^{2}$,

$$
\bar{T}=a T_{*}\left[1-\frac{1}{6}\left(e_{z}^{2}+e_{y}^{2}\right)\right],
$$

and in addition to the monopole, there is only the quadrupole

$$
a_{20}=\frac{1}{3} \sqrt{\frac{\pi}{5}}\left(2 e_{z}^{2}-e_{y}^{2}\right), \quad a_{21}=a_{2-1}=0, \quad a_{22}=a_{2-2}=-\sqrt{\frac{\pi}{30}} e_{y}^{2}
$$

which implies

$$
Q_{2}=\frac{2}{5 \sqrt{3}} \sqrt{e_{z}^{4}+e_{y}^{4}-e_{z}^{2} e_{y}^{2}} .
$$

The observed value of this is $Q_{2}(o b s) \approx 5.810^{-6}$, while the standard concordance model predicts $Q_{2}(i s o) \approx 1.298510^{-5}$. It has been suggested in previous works also that this discrepancy could be explained by an ellipsoidality of the universe [51,52]. This would require that the anisotropy of the background is suitably oriented with respect to the 
intrinsic quadrupole and cancels its power to sufficient amount. For any orientation then, we should have $Q_{2} \lesssim 2.720910^{-5}$ to be consistent with observations taking into account the cosmic variance. The constrains this implies on the dark energy equation of state parameters are rather tight.

One should, however, keep in mind that we have only studied the Bianchi I type background, which leaves a very simple pattern on the CMB, and assumed that to be somehow superposed with the anisotropies of the standard perturbed FLRW universe. This is of course a gross description. In a more realistic treatment, one should study the perturbations in matter in the anisotropic background. This is not a straightforward problem and will be tackled in section 5. We now focus only on the background anisotropy and assume that at least the order of magnitude of the limits one derives from it reflect correctly the properties of the universe.

In more general models, e.g. with time-varying $\delta$ and $\gamma$, one could allow more anisotropy. It is in principle possible for arbitrarily anisotropic expansion to escape detection from $\mathrm{CMB}$ as long as the expansion rates evolve in such a way that $e_{z}=e_{y}=0$. In other words, the quadrupole vanishes, if each scale factor has expanded - no matter how anisotropically - the same amount since the last scattering $\mathbf{\Upsilon}$.

Generating these effects at low redshift has an advantage that it relaxes constraints which would otherwise come from the CMB polarization [69] and could be strong for a given temperature anisotropy in isotropizing models because of the significant polarization anisotropy at last scattering. However, anisotropic dark energy could evade this since the optical depth to $z \sim 1$ is very small. More specifically, polarization of the CMB photons is generated by the Thomson scattering process on the free electrons. When the electron distribution has quadropole anisotropy, the scattered radiation will have net polarization. Thus it is clear that an anisotropy of the expansion will in principle always have imprints on the polarization pattern. However, most of the photons we see in the CMB were last scattered at $z \sim 1100$. Any model which predicts large anisotropies at such redshifts could thus easily be ruled out. At smaller redshifts, the effects of anisotropy on polarization are only secondary, since the photons do not scatter, but their energy distribution may be slightly modified. At the reionization epoch one expects again some photons to scatter, but the impact this has on CMB is restricted to the largest scales and suppressed by several orders magnitude. Thus it seems easier to explain the observed anomalies in the temperature spectrum without running into problems with unobserved anomalies in the polarization spectrum by anisotropies occurring in the later rather than in the earlier universe.

\subsection{SNIa Luminosities}

Anisotropy in the acceleration rates would be in principle seen as rotationally non-invariant luminosity distance - redshift relationships for the SNIa. The main CMB constraints on the

I We emphasize that this is only true for the CMB pattern due to the background anisotropy. In the next Section of the article we consider inhomogeneous perturbations, which in general have anisotropic signatures also in the case that they vanish at the background level. 
present model ensue from the quadrupole moment subject to effects of cosmic variance, and at least in specific models, the constraints can be loose or nonexistent. It is then interesting to see if one could limit the anisotropy with the present data on the nearby supernovae. More accurate SNIa data will become available in the near future, notably from the SNAP experiment ${ }^{+}$, but we find that useful constraints can already be derived. The luminosityredshift relationship of the SNIa can be used to probe the possible anisotropies in the expansion history. This is a complementary probe to the CMB quadrupole, since the SNIa objects are observed at the $z<2$ region, whereas CMB comes from much further away at $z \sim 1000$. The luminosity distance as a probe of anisotropies has been considered in $[72,73]$.

The spatial geodesic eq.(69) tells us that the direction $\hat{\mathbf{p}}$ of a photon that is coming towards us is constant. This reflects the orthogonality property of the Bianchi I model, in contrast to tilted models where the geodesics would involve rotation effects. For the null geodesics we have then

$$
-d t^{2}=\left(\hat{p}_{x}^{2} a^{2}+\hat{p}_{y}^{2} b^{2}+\hat{p}_{z}^{2} c^{2}\right) d r^{2}
$$

where $r^{2}=x^{2}+y^{2}+z^{2}$. The conformal distance is computed as $\int d r$. The luminosity distance at the redshift $z$ in the direction $\hat{p}$ is thus given by

$$
d_{L}(z, \hat{\mathbf{p}})=(1+z) \int_{t_{0}}^{t(z)} \frac{d t}{\sqrt{\hat{p}_{x}^{2} a^{2}+\hat{p}_{y}^{2} b^{2}+\hat{p}_{z}^{2} c^{2}}} .
$$

To test this prediction with the data, we apply the formula (72) for each observed redshift of a supernova and match its distance modulus $\mathcal{M}$ inferred from the observation to the one computed from Eq. (80) by

$$
\mathcal{M}=5 \log _{10}\left(\frac{d_{L}(z, \hat{\mathbf{p}})}{10 \mathrm{pc}}\right) .
$$

We have to then take into account also the angular coordinates of each individual supernovae in the sky which fix $\hat{\mathbf{p}}$ for each object. In our analysis we use the GOLD data set [74], which consists of five subsets of data*. We marginalize over the directions in the sky by integrating over the likelihood-weighted $\chi^{2}$ over three Euler angles and normalizing. Similarly we also marginalize over the present value of the Hubble constant. Taking these cosmologically irrelevant parameters into account makes the computations much heavier but is necessary. The results are summarized in Fig. 5. The best-fit anisotropic models are only slightly preferred over the $\Lambda$ CDM, the difference being $\Delta \chi^{2} \approx 1$. On the other hand, the SNIa data allows skewness in dark energy to an interesting degree.

In Fig. 6 we illustrate the SNIa constraints in the axisymmetric cases. Larger skewness $|\delta|$ would typically be compatible with the SNIa data for equations of state $w<-1$ and large matter densities. This means that even if the CMB formed isotropically at early time, it could be distorted by the acceleration of the later universe in such a way that it appears to us anomalous at the largest scales. The future SNIa data, with considerably

+ see http://snap.lbl.gov/.

* We find the angular coordinates of each of the 182 GOLD supernovae partly from [74] and [75] and from http://cfa-www.harvard.edu/ps/lists/Supernovae.html. 

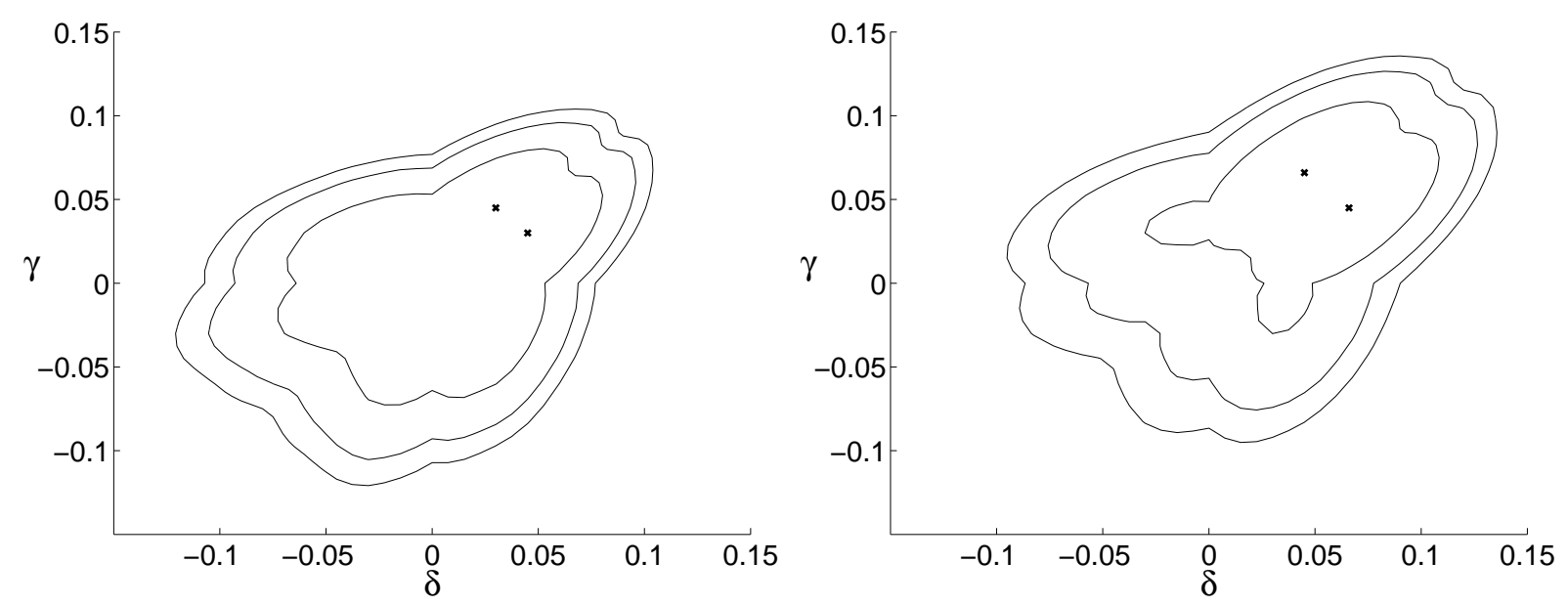

Figure 5. Limits on the skewness of dark energy arising from the SNIa data when $\Omega_{m}=0.3$. In the LHS, $w=-1$ and RHS $w=-1.2$. The contours correspond to $68.3,90$, and 95.4 percent confidence limits. The crosses mark the location of the best-fit models (there are two in each figure since, because we marginalize over the direction of the coordinates, there is a symmetry of projections about the $\delta+\gamma=0$ line).

improved error bars can be used to rule out this possibility, and to distinguish whether the possible statistical anisotropy was already there at last scattering or whether it is due to dark energy.

\section{Inhomogeneous Cosmology}

\subsection{Covariant Perturbations}

In the previous sections we have investigated the behaviour of an anisotropic universe which is homogeneous. Clearly this cannot be a realistic description of the universe, though it suffices as a good approximation when considering the expansion dynamics relevant to e.g. the supernovae luminosity distances. When considering e.g. the formation of galaxies and other structures in the universe, one has to allow perturbations about the homogeneous cosmological solution. Perko et al [76] pioneered the study of galaxy formation in anisotropic cosmologies. They discovered that in an anisotropic background, the gravitational waves can couple with the density modes. One of their results was that the shear can enhance the growth rate of matter perturbations. Later Bianchi I perturbations have been also considered within the coordinate approach to cosmological perturbations. The perturbation equations have been written quite explicitly in a gauge-ready form [77], including then more general sources. However, most studies have considered dust cosmologies $[78,79]$. In view on inflationary models, limiting matter sources to a slowly rolling scalar field is of course also of a particular interest. Indeed, very recently there have been studies of perturbations in anisotropic inflation which allow an anisotropically expanding background [80,81]. Considering late accelerating cosmologies, perturbation theory for elastic dark energy has been developed [82]. Yet this has only been done 

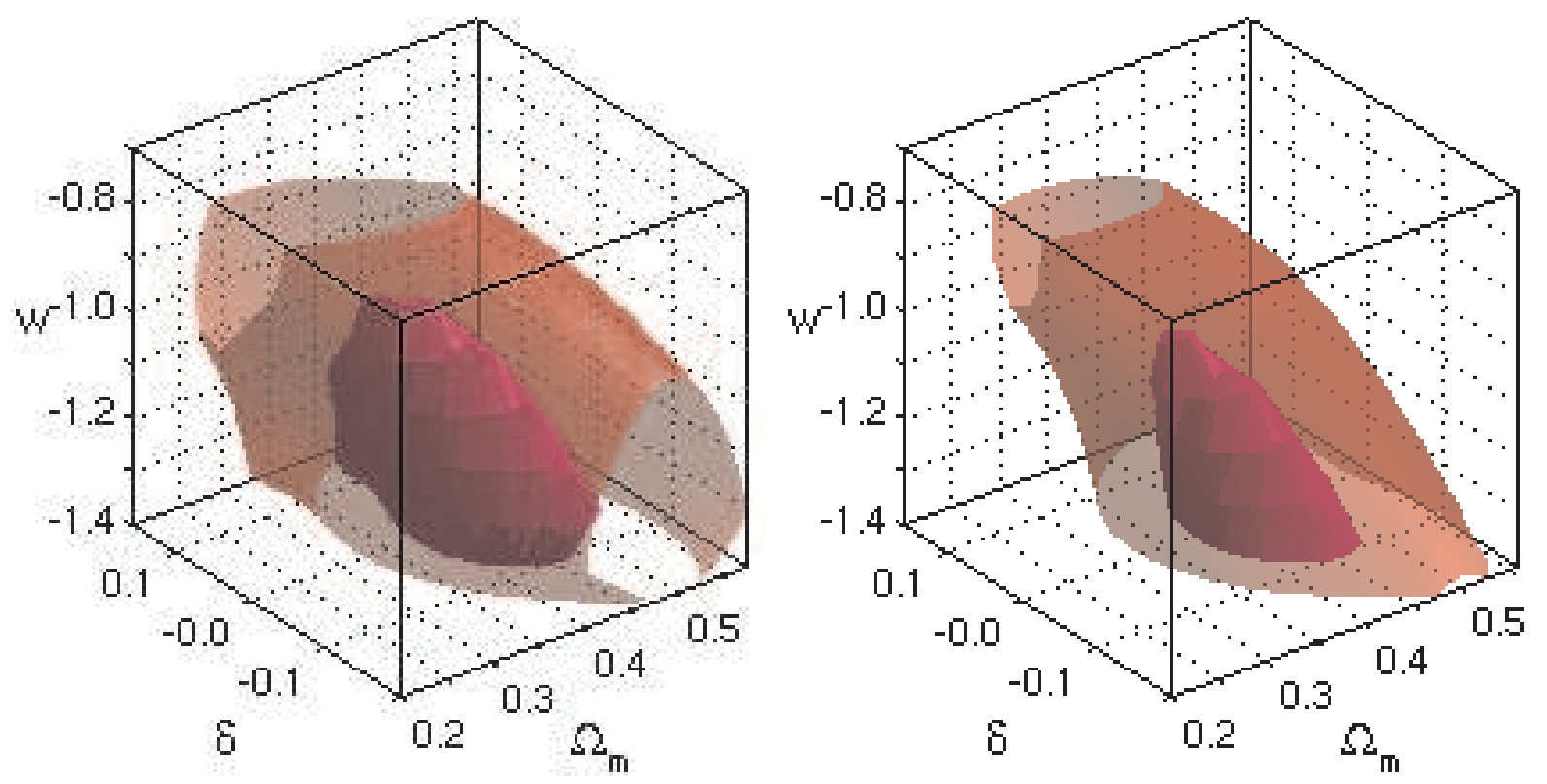

Figure 6. Constraints arising from the SNIa data in the axisymmetric case. Inside the darker isosurfaces, the fit is as good as in the $\Lambda$ CDM model, $\chi^{2}<158$. Inside the lighter shaded isosurfaces, one has $\Delta \chi^{2}<8.02$. In the left hand side figure, $\delta=\gamma$ and thus $w$ is the equation of state along the symmetry axis. In the right hand side figure, $\gamma=0$ and thus $w+\delta$ is the equation of state along the symmetry axis. One notes that larger skewness $\delta$ would typically be compatible with the SNIa data for "supernegative" equations of state $w<-1$ and large matter densities.

in the statistically isotropic framework, though the models would allow an anisotropic generalization [24]. In this paper we work within the covariant formalism. Therefore our notation follows most closely Dunsby's study of Bianchi I perturbations [83] in the covariant approach, which we slightly generalize from the point of view of possible non-isotropizing cosmologies, thus taking into account imperfect sources [84]. Maartens and Tsagas have considered imperfect sources in the form of magnetic fields in the covariant approach in detail $[85,86]$. We will omit most of the details of the covariant formalism and refer to, for instance the recent review of large-scale structure in relativistic cosmology discussing also more general spacetimes than the FLRW model [87].

5.1.1. Definitions. In a homogeneous background universe it is then convenient to consider variables which are defined as spatial gradients of some physically interesting quantities. These should then, by definition, count as perturbations. Since these gradients vanish in the background, the variables are by construction gauge-invariant. The basic variables we employ are defined as

$$
D_{a}=\frac{\ell}{\rho}{ }^{(3)} \nabla_{a} \rho, \quad Y_{a}={ }^{(3)} \nabla_{a} p, \quad Z_{a}=\ell^{(3)} \nabla_{a} \theta, \quad \Omega_{a}=\ell^{(3)} \nabla^{b} \omega_{a b}
$$


The first two of these are fluid quantities related to the energy density and pressure. $Z_{a}$ is the gradient of the expansion and $\Omega_{a}$ is the rotational perturbation.

In addition to these, we define some quantities related to the imperfect properties of the fluid:

$$
\begin{aligned}
& \Pi_{a}=h_{a}^{c} \pi_{c ; b}^{b}, \\
& \Xi_{a}=h^{b}{ }_{a}\left(\pi_{c d} \sigma^{c d}\right)_{; b}, \\
& \Sigma_{a}=\ell^{(3)} \nabla_{a} \sigma .
\end{aligned}
$$

It is also useful to define two scalars, the divergences of the acceleration and of the heat transfer, and two vectors, the spatial gradients of these as

$$
A=a_{; a}^{a}, \quad A_{a}={ }^{(3)} \nabla_{a} A, \quad Q=q_{; a}^{a}, \quad Q_{a}={ }^{(3)} \nabla_{a} Q .
$$

From these definitions it follows that

$$
{ }^{(3)} \nabla^{a} q_{a}=Q-a_{a} q^{a}, \quad{ }^{(3)} \nabla^{b} \pi_{a b}=\Pi_{a}-h_{a}{ }^{b} a^{c} \pi_{b c} .
$$

Note that our conventions are slightly different from the Tsagas et al [87], and when comparing the results the relations (87) are useful.

For a fluid with an equation of state $p=p(\rho, s)$, where $s$ is the entropy, we decompose the spatial gradient of the pressure as

$$
\ell Y_{a}=c_{s}^{2} \rho D_{a}+p E_{a}, \quad E_{a}=\frac{\ell}{p}\left(\frac{\partial p}{\partial s}\right){ }^{(3)} \nabla_{a} s,
$$

where the adiabatic sound speed is, at the zeroth order, related to the equation of state $w \equiv p / \rho$ by

$$
\dot{w}=\left(w-c_{s}^{2}\right)(1+w)(\theta+\xi) .
$$

We have defined an auxiliary variable $\xi$ to characterize the anisotropy,

$$
\xi \equiv \frac{\pi_{a b} \sigma^{a b}}{\rho(1+w)} .
$$

Using this variable will considerably simplify the following equations.

Note that the dot corresponds to the derivative along the flow as stated in Eq.(2). It corresponds to the derivative with respect to cosmic time $t$ only for scalars.

\subsubsection{Nonlinear Equations. The Raychaudhuri equation is}

$$
\dot{\theta}+\frac{1}{3} \theta^{2}+2\left(\sigma^{2}-\omega^{2}\right)-A+\frac{1}{2}(\rho+3 p)=0 .
$$

By projecting the covariant divergence of the stress-energy along and orthogonal to $u^{a}$, one obtains, respectively, the two conservation equations

$$
\begin{aligned}
& \dot{\rho}+(\rho+p) \theta+(1+w) \rho \xi+Q=0, \\
& (\rho+p) a_{a}+Y_{a}+\Pi_{a}+\mathcal{Q}_{a}=0,
\end{aligned}
$$

where we have defined

$$
\mathcal{Q}_{a}=\dot{q}_{a}+\sigma_{a}^{b} q_{b}+\frac{4}{3} \theta q_{a}
$$


The shear evolution equation can be written as

$$
\dot{\sigma}_{<a b>}-{ }^{(3)} \nabla_{<a} a_{b>}+\frac{2}{3} \theta \sigma_{a b}+\sigma_{c<a} \sigma_{b>}^{c}+E_{a b}+\frac{1}{2} \pi_{a b}=-a_{<a} a_{b>}+\omega_{<a} \omega_{b>} \cdot(95
$$

We will be able to drop away the RHS of this equation at the linearized level. The $<>$ means now the traceless symmetric par. $E_{a b}$ is the electric part of the Weyl tensor. This far our equations have been exact and thus very general but not very solvable in practice.

5.1.3. Linearized Evolution of Density. We now linearize the equations, supposing an anisotropic but homogeneous background. We assume that the heat flux and vorticity vanish at the zero order. All the possible spatial gradients vanish in the background, since it is homogeneous. However, we allow anisotropy both in the metric, $\sigma_{a b}$, and in the fluid $\pi_{a b}$. Note that in this subsection we do not yet specify the type anisotropy, and thus the following equations apply in any Bianchi type spacetime.

We find that the overdensity evolves as

$$
\dot{D}_{a}=w \theta D_{a}-(1+w) Z_{a}-\sigma_{a}^{c} D_{c}+(1+w) \xi\left(D_{a}-\ell a_{a}\right)+\frac{\ell}{\rho}\left(\theta \Pi_{a}-\Xi_{a}\right)+\frac{\ell}{\rho}\left(\theta \mathcal{Q}_{a}-Q_{a}\right)
$$

where $w \equiv p / \rho$. The evolution equation for the gradient of the expansion scalar is

$$
\dot{Z}_{a}=-\frac{1}{2} \rho D_{a}-\frac{2}{3} \theta Z_{a}-\sigma_{a}^{c} Z_{c}-3 \ell \sigma^{2} a_{a}+\ell A_{a}-4 \sigma \Sigma_{a}+\frac{3}{2} \ell \Pi_{a}+\frac{3}{2} \ell \mathcal{Q}_{a}
$$

From the continuity equation (93) we see that $A_{a}$, as defined in (86), may be given as

$$
A_{a}=-\frac{1}{\rho(1+w)}\left({ }^{(3)} \nabla^{2} Y_{a}+{ }^{(3)} \nabla_{a}{ }^{(3)} \nabla^{b(3)} \nabla^{c} \pi_{b c}+{ }^{(3)} \nabla_{a}{ }^{(3)} \nabla^{b} \mathcal{Q}_{b}\right)-2 c_{s}^{2} \theta \Omega_{a}
$$

where one can show that

$$
{ }^{(3)} \nabla_{a}{ }^{(3)} \nabla^{b} \mathcal{Q}_{b}=\left({ }^{(3)} \nabla_{a} Q\right)^{\bullet}+2 \theta^{(3)} \nabla_{a} Q+\sigma^{b}{ }_{a}^{(3)} \nabla_{b} Q+2 \sigma^{b c(3)} \nabla_{a}{ }^{(3)} \nabla_{b} q_{c} .
$$

The evolution equation for the density gradients follows then by differentiating the first order equation and using all the previous formulas. We find

$$
\begin{aligned}
\ddot{D}_{a}+ & {\left[\left(\frac{2}{3}+c_{s}^{2}-2 w\right) \theta-(1+2 w) \xi\right] \dot{D}_{a}+2 \sigma_{a}{ }^{b} \dot{D}_{b} } \\
& -\left[\left(\frac{1}{2}+4 w-\frac{3}{2} w^{2}-3 c_{s}^{2}\right) \rho+\left(2 w-6 c_{s}^{2}\right) \sigma^{2}+c_{s}^{2(3)} \nabla^{2}\right] D_{a} \\
& +\left[\left(\frac{2}{3}-2 w+c_{s}^{2}\right) \theta-(1+2 w) \xi\right] \sigma_{a}^{b} D_{b}+\sigma_{a}^{b} \sigma_{b}{ }^{c} D_{c}-\pi_{a}{ }^{b} D_{b} \\
& -\left[\left(1+c_{s}^{2}+w\right) \dot{\xi}+\left(\dot{c}_{s}^{2}+\frac{1}{3} \theta\left(2+c_{s}^{2}\left(-1+3 c_{s}^{2}-3 w\right)+5 w\right)\right) \xi\right. \\
& \left.+c_{s}^{2}\left(c_{s}^{2}-w\right) \xi^{2}\right] D_{a}-\left[w\left({ }^{(3)} \nabla^{2}-3 \sigma^{2}+\dot{\xi}\right)+\left(\frac{5}{3} w-c_{s}^{2}\right) \theta \xi+\left(w-c_{s}^{2}\right) \xi^{2}\right] E_{a} \\
& -w \xi\left(\dot{E}_{a}+\sigma_{a}{ }^{b} E_{b}\right)+2(1+w) c_{s}^{2} \theta \Omega_{a}-4 \sigma(1+w) \Sigma_{a}=S_{a}^{(q)}+S_{a}^{(\Pi)} .
\end{aligned}
$$


The right hand side of Eq.(98) vanishes if we consider a case that the imperfect perturbations can be neglected. The sources are due to the heat flux perturbations as

$$
\begin{aligned}
\frac{\rho S_{a}^{(q)}}{\ell} & =-\dot{Q}_{a}-\left[\left(2+c_{s}^{2}\right) \theta+\left(1+c_{s}^{2}\right) \xi^{2}\right] Q_{a}-\sigma_{a}{ }^{b} Q_{b} \\
& +{ }^{(3)} \nabla_{a}{ }^{(3)} \nabla^{b} \mathcal{Q}_{b}+(\theta+\xi) \dot{\mathcal{Q}}_{a}+\xi \sigma_{a}{ }^{b} \mathcal{Q}_{b} \\
& +\left[(3 w-2) \sigma^{2}+\rho+\dot{\xi}+\left(3+c_{s}^{2}+w\right) \theta \xi+\left(1+c_{s}^{2}\right) \xi^{2}\right] \mathcal{Q}_{a}
\end{aligned}
$$

The sources from perturbations of the anisotropic stress are the following

$$
\begin{aligned}
\frac{\rho S_{a}^{(\Pi)}}{\ell} & ={ }^{(3)} \nabla_{a}{ }^{(3)} \nabla^{b(3)} \nabla^{c} \pi_{b c}-\dot{\Xi}_{a}-\left[\left(\frac{4}{3}+w\right) \theta+(1+w) \xi\right] \Xi_{a} \\
& +(\theta+\xi)\left(\dot{\Pi}_{a}+\sigma_{a}{ }^{b} \Pi_{b}\right) \\
& +\left[3\left(1-w+c_{s}^{2}\right) \rho+3 c_{s}^{2} \sigma^{2}+\dot{\xi}+\left(3+2 c_{s}^{2}\right) \theta \xi+\left(1+c_{s}^{2}\right) \xi^{2}\right] \Pi_{a} .
\end{aligned}
$$

We cannot derive the general evolution equations for these terms. Their behaviour depends completely on the nature of the fluid. In fact this is true also for the other quantities, but then we have a simpler and conventional way of parameterizing the unspecified properties in terms of $w, c_{s}^{2}$.

5.1.4. Linearized Evolution of Shear. In addition to the unspecified matter part, the shear $\sigma$ and its gradient $\Sigma_{a}$ appear in our evolution equations. So we should find a way to solve for them too. From the shear evolution equations (95) we easily find that now

$$
\dot{\sigma}=\frac{1}{\sigma}\left[\frac{1}{2} \sigma^{a b(3)} \nabla_{(a} a_{b)}-\frac{1}{2} \sigma^{a b} \sigma_{a f} \sigma_{b}^{f}-\frac{2}{3} \theta \sigma^{2}-\frac{1}{2} \sigma^{a b}\left(E_{a b}-\frac{1}{2} \pi_{a b}\right)\right] .
$$

From the definition of $\Sigma_{a}$, and the commutation rules for the spatial and time derivatives, one gets that $\dot{\Sigma}_{a}=\frac{1}{3} \theta \Sigma_{a}+\ell^{(3)} \nabla_{a} \dot{\sigma}-\ell h^{b}{ }_{a}\left(\sigma_{; c} u^{c}\right)_{; b}+\ell h^{d}{ }_{a}\left(h^{b}{ }_{d} \sigma_{; b}\right)_{; c} u^{c}$. Inserting the result (101) and linearizing then gives

$$
\begin{aligned}
\dot{\Sigma}_{a} & +\frac{1}{\sigma^{2}}\left[\frac{1}{2} \sigma^{b c} \sigma_{b f} \sigma_{c}^{f}+\frac{2}{3} \theta \sigma^{2}+\frac{1}{2} \sigma^{b c}\left(E_{b c}-\frac{1}{2} \pi_{b c}\right)\right] \Sigma_{a} \\
& +\frac{1}{\sigma}{ }^{(3)} \nabla_{a}\left[\frac{1}{2} \sigma^{c d(3)} \nabla_{(c} a_{d)}-\frac{1}{2} \sigma^{c d} \sigma_{c f} \sigma^{f}{ }_{d}-\frac{2}{3} \theta \sigma^{2}-\frac{1}{2} \sigma^{c d}\left(E_{c d}-\frac{1}{2} \pi_{c d}\right)\right] \\
& +\sigma_{a}^{c}{ }_{a} \Sigma_{c}+\ell \theta \sigma a_{a}=0 .
\end{aligned}
$$

However, it is also possible to solve the shear away without evolving it in time but relating it to the other variables. This is because the time-space components of the Einstein field equations yield three additional constraint equations which we have not exploited this far. These constraints can be put in the form

$$
\Sigma_{a}=\frac{2}{3 \ell} Z_{a}+\Omega_{a}+\sigma_{a}^{c} a_{c}
$$

As a consistency check, one might take the time derivative of this equation and see whether it is compatible with the evolution equation for the shear we just wrote down in (102). It turns out that this constraint is indeed preserved in time. 
We can use this information to eliminate $\Sigma_{a}$ from the matter evolution equation (98). In terms of the quantities appearing in that equation, we have

$$
\begin{aligned}
(1+w) \Sigma_{a} & =-\frac{2}{3} \dot{D}_{a}+\frac{2}{3}\left[\theta+\left(1+c_{s}^{2}+w\right) \xi\right] D_{a}+\left(c_{s}^{2}-\frac{2}{3}\right) \sigma_{a}{ }^{b} D_{b} \\
& +w\left(\frac{1}{3} \xi E_{a}-\sigma_{a}{ }^{b} E_{b}\right)+(1+w) \frac{1}{\rho} \Omega_{a} \\
& +\frac{\ell}{\rho}\left[\frac{2}{3}(\theta+\xi) \Pi_{a}-\sigma_{a}{ }^{b} \Pi_{b}+\frac{2}{3}\left(\xi \mathcal{Q}_{a}-Q_{a}\right)-\sigma_{a}{ }^{b} \mathcal{Q}_{b}\right] .
\end{aligned}
$$

We note that $\Sigma_{a}$ does of course not vanish even in the general statistically isotropic case.

\subsection{Structure Formation}

In the case of magnetic fields it has been learned that neglecting the background anisotropy and resorting to an almost-FLRW treatment can be a good approximation on large scales when the magnetic field is weak, but the shear may introduce small-scale effects which are accurately captured only by the Bianchi I treatment [86]. As we consider a general anisotropic fluid not necessarily reducible to magnetic field plus perfect matter, it is thus interesting to study under which conditions we can safely regard the FLRW description as accurate, and under which conditions the background anisotropies should be taken into account in order not to miss possible new physical effects.

In the following, we write down the general equations of the last sections in three cases. To close the system of equations, one must specify the anisotropic properties of the fluid. In subsection (5.2.2) we assume and study the case that the anisotropy is small. Then, it turns out, the almost-FLRW approach is valid, with however some direction-dependence of the perturbations. In subsections (5.2.3) and (5.2.4) we do not assume that the anisotropies are negligibly small at the background order, but make other simple assumptions about the properties of perturbations to fully specify the system. In these cases, the shear effects could not be described by the almost-FLRW treatment.

5.2.1. Scalar Equations. Since the matter density can be described as a scalar field, it is usual to concentrate on the scalar modes of perturbations when studying linear evolution in cosmology. To make contact with these studies, it is useful to extract the scalar part of the more general equations in the previous section. A conventional procedure for this is based on a local decomposition [88] of the spatial gradients,

$$
\ell \equiv{ }^{(3)} \nabla^{a} X_{a}=\frac{1}{3} h_{a b} X+\left[X_{(a b)}-\frac{1}{3} h_{a b} X\right]+X_{[a b]} .
$$

As an example, we may act on the density gradient of matter field, $D_{a}$, with ${ }^{(3)} \nabla^{a}$, to get the spherically symmetric part of the scalar perturbation. Explicitly, we define this variable as

$$
\ell^{(3)} \nabla^{a} D_{a} \equiv \Delta .
$$


In the presence of shear there arise other relevant scalars quantifying the properties of matter distribution. We define them as follows

$$
\begin{array}{ll}
\ell \sigma^{a b(3)} \nabla_{a} D_{b} & \equiv \Delta^{\sigma}, \\
\ell \pi^{a b(3)} \nabla_{a} D_{b} & \equiv \Delta^{\pi}, \\
\ell \sigma^{a c} \sigma_{c}^{b(3)} \nabla_{a} D_{b} & \equiv \Delta^{\sigma^{2}} .
\end{array}
$$

It then follows that

$$
\begin{aligned}
& \ell^{(3)} \nabla^{a} \dot{D}_{a}=\dot{\Delta}+\Delta^{\sigma}, \\
& \ell^{(3)} \nabla^{a} \ddot{D}_{a}=\ddot{\Delta}+2 \dot{\Delta}^{\sigma}+\theta \Delta^{\sigma}+\Delta^{\sigma^{2}}-\Delta^{\pi}, \\
& \ell^{(3)} \nabla^{a} \sigma_{a}{ }^{b} \dot{D}_{b}=\dot{\Delta}^{\sigma}+\theta \Delta^{\sigma}+\Delta^{\sigma^{2}}-\Delta^{\pi} .
\end{aligned}
$$

In a completely analogous way, one may derive scalar variables from other gradients appearing in our considerations. The scalar equations one then derives by acting on the general equations with $\ell^{(3)} \nabla^{a}$. It is straightforward to pick up the new coefficients of the scalar variables by using the definitions (106-109) and the identities (110-112).

In the most general case, the system is very complicated. In the following, we will comment the physical implications under three different approximations and the motivations for such approximations: I) The case that the anisotropy is small and can be treated as perturbation, II) the case that the from of anisotropy perturbation is determined by it's background form III) the case that the the anisotropy is homogeneous also at the perturbative level.

5.2.2. Case I: Small Anisotropy. We make the simplifying assumption that the anisotropy is small. This is justified by the constraints on the late-time anisotropies arising from the quadrupole pattern in the CMB and the directional dependence of the luminositydistance. Thus we regard both the metric $\sigma$ and the fluid $\pi$ as first order quantities in the perturbative equations. Then we are allowed to drop a considerable number of terms from the equations, but the largest effects from anisotropy are still taken into account since some first order terms remain. Note that would be absent or of a different form in the FLRW case. Thus this seems as a very reasonable starting point for the first analysis of the structure formation with an imperfect dark energy scenario.

With this assumption the Eq.(98) reduces to

$$
\begin{aligned}
\ddot{D}_{a} & +\left(\frac{2}{3}+c_{s}^{2}-2 w\right) \theta \dot{D}_{a}+\left[\left(\frac{1}{2}+4 w-\frac{3}{2} w^{2}-3 c_{s}^{2}\right) \rho+c_{s}^{2(3)} \nabla^{2}\right] D_{a} \\
& =-w^{(3)} \nabla^{2} E_{a}-2(1+w) c_{s}^{2} \theta \Omega_{a}+S_{a}^{(\Pi)}
\end{aligned}
$$

where the imperfect fluid terms are given as

$$
S_{a}^{(\Pi)}=\frac{\ell}{\rho}\left({ }^{(3)} \nabla_{a}{ }^{(3)} \nabla^{b(3)} \nabla^{c} \pi_{b c}+\theta \dot{\Pi}_{a}\right)+3 \ell\left(1-w+c_{s}^{2}\right) \Pi_{a} .
$$

Directly from the metric, the anisotropic terms would contribute only quadratically. From the fluid they contribute also at the first order affecting then directly the evolution of the density perturbation as the source term (114). 
By extracting the scalar part of this system as described in subsection (5.2.1), one may verify that the result is equivalent to the evolution equation for generalized matter when imperfect fluid terms are taken into account [89]. One thus arrives at the conclusion that the law governing the evolution of overdensities is exactly the same as in an isotropic case when we have neglected the anisotropy terms at the background order. However, one should be careful to not deduce that this means that the total evolution is isotropic in such a case. The system Eq.(113) presents three equations for the time evolution of three independent spatial gradients of the density, and in the general case they could have both different solutions and different forms. In particular, this could be a consequence of an intrinsic anisotropic stress source, $\Pi_{a}$, which could be zero for a given direction $a$ but not for the others. Our formula Eq.(113) thus provides a tool to study fully consistently and covariantly the fundamentally anisotropic properties of matter inhomogeneities in cosmology, which may be hidden in the standard description of the overdensity scalar perturbation. One may witness the anisotropy by monitoring the solutions of the individual equations of the system (113), while summing their gradients yields the scalar quantity which has a straightforward interpretation as the overdensity in the standard FLRW cosmology, which at small scales does not depend on the gauge choice.

This also shows that, even when a Bianchi universe is close to the FLRW model, one cannot consider the anisotropies in the perturbed Bianchi universe as a sum of FLRW perturbations and the small anisotropies of the exact Bianchi model. When an imperfect source is present, its perturbations (114) can break explicitly the statistical isotropy already at the linear level. These fluid perturbations of course couple to all other perturbations in the metric and in matter. These become then statistically anisotropic as well. Therefore the fluctuations of the model evolve differently than in a FLRW model, in particular the resulting anisotropy pattern is not a simple superposition of standard isotropic perturbations and a Bianchi type perturbation about the FLRW. However, this kind of approximation could be reasonably good when only perfect fluid sources are present. This is because the shear in the metric typically enters the equations only trough $\sigma^{2}$, i.e. quadratically. In such a case, one could expect that some effects are given, to first perturbative order, as the sum of ordinary FLRW perturbation and the Bianchi pattern.

We conclude that when the anisotropy is small, perturbations in each direction behave like in different isotropic universes, each obeying their Eq.(113).

5.2.3. Case II: "Adiabatic" Anisotropy. Clearly, to perform actual calculations one has to specify the properties of the fluids under consideration. We would still like to keep the discussion general, and therefore we apply a parametrization which could approximate a wide range of different models at a certain limit. We then choose the $w, \delta, \gamma$ parametrization as it seems a natural and straightforward generalization of the concept of equation of state in the FLRW universe. In the Bianchi I background, one may fully specify the properties of a fluid with energy density $\rho$ by giving the three pressures in different directions, or equivalently, the three equations of state for the fluid. In the following, we assume this holds to the perturbed order. In principle the inhomogeneities in the stresses would bring additional degrees of freedom, but our parametrization assumes they are described by the 
homogeneous equations of state. This may be interpreted as a generalized adiabaticity condition, since in absence of entropy, the pressure perturbation evolution is determined by the homogeneous background evolution.

Recall the decomposition of the fluid content. In Eq.(15) we described the energy momentum tensor for the anisotropic component. It may be written as

$$
T_{X \nu}^{\mu}=\operatorname{diag}\left(-1, w_{X}-\delta-\gamma, w_{X}+2 \delta-\gamma, w_{X}-\delta+2 \gamma\right) \hat{\rho}_{X}
$$

We are now in a frame having $u^{\mu}=(1,0,0,0)$. Therefore, the components in the stressenergy tensor (6) could now be identified as

$$
\begin{aligned}
& \rho=\hat{\rho}_{X}, \quad p=w_{X} \rho_{X}, \quad q_{a}=0, \\
& \pi_{b}^{a}=\operatorname{diag}(0,-\delta-\gamma, 2 \delta-\gamma,-\delta+2 \gamma) \rho_{X} .
\end{aligned}
$$

From the observation that $q_{a}$ vanishes one could justify to use the equation (98) with the heat flux sources $S^{(Q)}$ turned to zero. If the anisotropy is to be small, the condition is clearly that $\gamma$ and $\delta$ are small. One finds that the gradients of the anisotropic stresses are now

$$
\Pi_{a}=\frac{\rho}{\ell}\left[0,-(\delta+\gamma) D_{x},(2 \delta-\gamma) D_{y},(-\delta+2 \gamma) D_{z}\right]
$$

and the sum term appearing in the source term Eq.(100) is then

$$
\begin{aligned}
\frac{\ell^{(3)} \nabla_{a}{ }^{(3)} \nabla^{b(3)} \nabla^{c} \pi_{b c}}{} & =-(\delta+\gamma)^{(3)} \nabla_{a}{ }^{(3)} \nabla^{x} D_{x} \\
& +(2 \delta-\gamma)^{(3)} \nabla_{a}{ }^{(3)} \nabla^{y} D_{y}-(\delta-2 \gamma)^{(3)} \nabla_{a}{ }^{(3)} \nabla^{z} D_{z} .
\end{aligned}
$$

The latter source term thus couples to $D_{b}$-terms with $a \neq b$ into the evolution equation for $D_{a}$, (98). Since the metric shear $\sigma^{2}=\frac{1}{27}\left(R^{2}+S^{2}-R S\right) \theta^{2}$ clearly the small anisotropy limit corresponds to $R, S \ll 1$.

Let us be more precise about this limit. One observes that if the coefficients $\delta$ and $\gamma$ are supposed to be perturbatively small, all contributions to the perturbations equations at the linear order vanish. This is because we consider that the constant coefficient of the homogeneous anisotropy gives the relation between the density gradient and the (anisotropic) pressure gradient as well as the relation between the background density and the homogeneous (anisotropic) pressure. Since the density gradient is already a perturbation, the anisotropic pressure gradients (115) should be of the second order in perturbations. Similarly with the metric shear: if $S$ and $R$ are regarded as first order, then all the terms involving $\sigma$ in the evolution equations are higher than first order. Therefore the anisotropic terms would decouple from all perturbations equations and the effect of anisotropy would indeed be negligible. Note that the homogeneous anisotropy and the gradient of the anisotropy could be both of the linear order, though it is not the case with the present parametrization. Now the simplest nontrivial corrections to the standard FLRW treatment appear if one considers the squares of the anisotropy variables as first order perturbations. 
In general, if the anisotropy terms satisfy such conditions, the system of three equations for the gradients of the matter aggregations can be written as

$$
\begin{aligned}
\ddot{D}_{a} & +\left(\frac{2}{3}+c_{s}^{2}-2 w\right) \dot{D}_{a}+2 \sigma_{a}{ }^{b} \dot{D}_{b}-\left(\frac{1}{2}+4 w-\frac{3}{2} w^{2}-3 c_{s}^{2}\right) \rho D_{a} \\
& -\left(\frac{1}{3}+2 w-c_{s}^{2}\right) \theta \sigma_{a}{ }^{b} D_{b}-{ }^{(3)} \nabla^{2}\left(c_{s}^{2} D_{a}+w E_{a}\right)+2(1+w) c_{s}^{2} \theta \Omega_{a} \\
& =4 \sigma(1+w) \Sigma_{a}+\frac{\ell}{\rho}\left[{ }^{(3)} \nabla_{a}{ }^{(3)} \nabla^{b(3)} \nabla^{c} \pi_{b c}-\dot{\Xi}_{a}-\left(\frac{4}{3}+w\right) \theta \Xi_{a}\right. \\
& \left.+\theta \dot{\Pi}_{a}+3\left(1-w+c_{s}^{2}\right) \rho \Pi_{a}\right] .
\end{aligned}
$$

Now, in addition to the imperfect source stresses, the coupling of the metric shear will generate anisotropies in the perturbations.

We conclude that there exists a generalization of the adiabatic condition to Bianchi I universes which then uniquely determine the perturbative stresses of the fluid.

5.2.4. Case III: Smooth Anisotropy. Yet one more physically motivated approximation is to consider the anisotropy field as smooth. A dark energy -like component in usual cases is considered to be rather smooth, especially at small scales. In fact no evidence for clumping of dark energy has been found. Slowly rolling light fields with minimal couplings tend to have negligible perturbations. For the cosmological constant this becomes an exact covariant statement: there are no cosmological fluctuations in the usual $\Lambda$-term. When the pressure varies in direction and with time, its spatial gradients may be only approximately zero. Basically, one then considers the effects of the anisotropic background expansion on the matter inhomogeneities. To do this consistently one should couple not only the metric $\sigma$ but also the fluid $\pi$ anisotropy to the equations. Then we can, however, neglect the possible entropy in the perturbations by a direct analogy with the usual $\Lambda$ term. One notes that the entropy couples also to the large scale perturbations through the anisotropic terms, whereas in an FLRW universe one may neglect $E$ in the limit ${ }^{(3)} \nabla^{2} \rightarrow 0$.

Indeed, for the model of subsection (5.2.2), one could consider a very tidy set of perturbation equations where the isotropic perturbations of the $\Lambda$-term identically vanish, and the condition $\xi=0$ allows to drop a large number of terms. For the variant of the model, discussed in subsection 3.2.2, the isotropic pressure of the $\Lambda$-term is not constant and therefore cannot be smooth. This introduces entropy between the $\Lambda$-term and matter. One would then consider the evolution of the total density gradient $D_{a}$, as that is simply related to the matter density gradient $D_{a}^{(m)}$ by $D_{a}^{(m)}=(1-U) D_{a}$. The anisotropic stress and the metric shear at the background level would then be

$$
\begin{aligned}
\pi_{b}^{a} & =\operatorname{diag}[0,-1,2,-1] \delta \rho_{\Lambda} \\
\sigma_{b}^{a} & =\frac{1}{3} \operatorname{diag}[0,1,-2,1] H R,
\end{aligned}
$$

giving then $\sigma^{2}=(H R)^{2} / 3$. Note that $\pi_{b}^{a}$ is constant (when $\delta$ is), and thus its fluctuations $\Pi_{a}$ vanish. However, similar conclusion does not hold for the $\sigma$-terms: the metric shear has fluctuations and generally $\Sigma_{a}$ is nonzero. Therefore in the model with the constant 
anisotropic pressure field generates effects on the first order fluctuations. In fact this is already reflected in the fact that the total sound speed squared of the system (61) can become nonzero when $\delta$ is nonzero. Because there is entropy present, the possible negativity of the sound speed does not necessarily lead to instabilities. These effects could be used to constrain further the scenario proposed in subsection 3.2.2.

We conclude that either the isotropic or the anisotropic pressure of an imperfect fluid always fluctuates.

\section{Conclusions and Discussion}

We investigated the possibility that the accelerated expansion of our universe, both during the early inflation and the present dark energy domination, is driven by a component with an anisotropic equation of state. The motivation for this investigation comes from the frequent appearance vector fields and possible Lorentz violations in fundamental theories, from the need to the test the robustness of the basic assumptions of cosmology, and from the hints of statistical anisotropy of our universe that several observations seem to suggest. From the examination of the background dynamics we learned that

- There exist (anisotropic) scaling solutions even without coupling between the components.

- An anisotropic generalization of the $\Lambda$-term has nontrivial dynamics given if its isotropic pressure is time-varying.

- The quadropole constraint on the anisotropy may be avoided, and the present SNIa data allows large anisotropy.

From the analysis of system of perturbations the following was found

- Even in the limit of weak anisotropy, the linearized perturbations will be directiondependent.

- The linearized anisotropy is the sum of FLRW perturbations and Bianchi I background only for perfect-fluid cosmology.

- Either in the limit of vanishing entropy or in the limit of homogeneous field the almost FLRW treatment is not valid.

Let us elaborate on these and other results.

We started by presenting a phenomenological description of a such an anisotropy in terms of the skewness parameters $\delta$ and $\gamma$, and derived the basic equations describing the model as a dynamical system, and obtained the generic asymptotic evolution of the universe in some simple cases. Several scaling solutions were found both within the matter dominated epoch as well as during an accelerated expansion phase (with the summary of fixed points presented in the Table 2). This seems to be interesting, since while in the FLRW universe it has been proven difficult to address the coincidence problem by finding a model entering from a matter-dominated scaling solution to an accelerating scaling solution, allowing for the presence of three expansion rates now opens up the 
possibility of describing a universe entering from a perfect fluid dominated scaling to an anisotropically accelerating scaling era, which might eventually help to understand the coincidence problem. In that framework, it is easier to find models with natural parameter values avoiding the fine-tuning problems of cosmological constant or scalar field quintessence [20, 48, 90].

We also contemplated upon the possibility of an anisotropic generalization of the cosmological constant constant term. Interestingly, we found that a model with a constant density and constant anisotropic pressures $\left(\rho=\rho_{0}, \delta=\delta_{0}, \gamma=\gamma_{0}\right)$ is indeed a consistent covariant modification of the Einstein's cosmological term which could generate a viable expansion history (with small enough $\delta_{0}$ and $\gamma_{0}$ ) that exhibits nontrivial anisotropic properties. We mentioned noncommutative properties of spacetime as a possible origin of anisotropies in a cosmological $\Lambda$-term. One could consider different motivations by writing the vacuum energy as an integral of the zero-point energies over the $\mathbf{k}$ up to some cut-off (summed over every possible field). If there were any isotropy-breaking physical principle which allowed only wavevectors with specific directions, the vacuum energy would acquire nonzero $\delta$ and $\gamma$. More specifically, if the cut-off depended not only on the magnitude $k$ of the wavevector $\mathbf{k}$, but also on its direction (i.e. some directions would be excluded from the integral or contribute with less weight to the vacuum energy) this could explain both the smallness of the observed cosmological constant and the apparent anisotropic features in the cosmological data.

The amount of possible anisotropy, as described by the skewness parameters, can be constrained by the $\mathrm{CMB}$ and SNIa observations. We found that the present CMB data constraints can be much tighter than the constraints from the anisotropies in the luminosity-distance -redshift relationship of SNIa. However, there are classes of models where the CMB bounds from the background quadrupole anisotropy can be avoided altogether while there is significant anisotropy at late times that could be observed in the late SNIa distributions and which could leave statistically anisotropic imprints at the large-angle CMB. We point out, however, that in more general models, e.g. with timevarying $\delta$ and $\gamma$, one could allow more anisotropy. It is in principle possible for an arbitrarily anisotropic expansion to escape detection from CMB (considering only the effects from the background), as long as the expansion rates evolve in such a way that $e_{z}=e_{y}=0$. In other words, the (background) quadrupole vanishes, if each scale factor has expanded - no matter how anisotropically - the same amount since the last scattering.

We also considered the inhomogeneous cosmology of these models. The covariant formalism was employed to derive the general evolution equations for the perturbations in a universe with background anisotropies, i.e. imperfect fluid terms and shear of the metric. Our formalism and equations thus provide a tool to study fully consistently and covariantly the fundamentally anisotropic properties of matter inhomogeneities in cosmology, which may be hidden in the standard description of the overdensity scalar perturbation. One may witness the anisotropy by monitoring the solutions of the individual equations of the system (113), while summing their gradients yields the scalar quantity which has a straightforward interpretation as the overdensity in the standard FLRW cosmology, which at small scales does not depend on the gauge choice. 
The implications of anisotropic sources for the formation of structures were discussed in several limiting cases. In particular, in the cases examined, the deviations from isotropy are small enough that one may treat all the imperfect terms as first order perturbations. We noticed that this gives a result which is not obtainable by a straightforward generalization of the usual FLRW equations. If one considers the evolution equation of matter perturbations (in its closed form (113)), one notices that while the metric shear would enter only at the second order, the imperfect fluid terms are present at the first order level. Thus, even when the Bianchi universe would be close to the FLRW model, one cannot consider the anisotropies in the perturbed Bianchi universe as a sum of FLRW perturbations and the small anisotropies of the exact Bianchi model. This is seen from the fact that when an imperfect source is present, its perturbations (114) can break explicitly the statistical isotropy already at the linear level. In general, the perturbation structure of these models allows a rich array of possibilities. The anisotropic properties seem to usually extend to the smaller scales also, but this feature is model-dependent and the constraints it implies must be studied on a case-by-case basis.

The present SNIa data allows anisotropic acceleration, but future SNAP data could set things straight about the skewness of dark energy and so of its nature. Such possibility would open a completely new window not only on the nature of the CMB anomalies but also into high energy physics models beyond the usual isotropic candidates of inflation or dark energy models such as the inflaton, the quintessence scalar fields or even an isotropic cosmological constant. Hence, an anisotropic equation of state may not only successfully unify the early inflation with the present days acceleration, but might also be the culprit for both the apparent problem of cosmic coincidence as well as the large-angle anomalies in the CMB.

The detailed signatures of the anisotropies, either originating from the early or the late accelerating epoch of the universe, to the full sky maps of the CMB should be determined: could these signatures rule some or all of the models out, or could they in fact be identified with the observed anomalies? Investigations along these lines are ongoing.

\section{Appendix: Pedestrian notation for Bianchi I}

In this Appendix we review our system within the coordinate formalism. Our main developments in the bulk of the paper follow the covariant approach for the sake of homogeneity. However, for some considerations the full covariant machinery is unnecessary, and analysis is simpler and perhaps more transparent by specifying the coordinate system and writing the equations in terms of the metric components.

The Bianchi I metric as generalization of the flat FLRW metric is

$$
d s^{2}=-d t^{2}+a^{2}(t) d x^{2}+b^{2}(t) d y^{2}+c^{2}(t) d z^{2} .
$$

Due to the presence of three scale factors $a, b$ and $c$, one has to introduce also three expansion rates. In principle all these could be different, and in the limiting case that all of them are equal, one of course recovers the FLRW case. Note that we use the roman letters to label the covariant indices, like in the expression for the energy momentum tensor 
(6), whereas we use the Greek letters to label the indices of tensors when we refer to the components in the specific choice of basis (122). The perfect fluid energy momentum tensor is then simply

$$
T_{(m) \nu}^{\mu}=\operatorname{diag}\left(-1, w_{m}, w_{m}, w_{m}\right) \rho_{m},
$$

In the coordinate system we have chosen, the energy-momentum tensor must be diagonal, since the nondiagonal Einstein tensor vanishes identically. However, the pressure in each spatial direction could be different. We then parametrize the anisotropy of the pressure by the two skewness parameters

$$
3 \delta \equiv\left(p_{y}-p_{x}\right) / \rho, \quad 3 \gamma \equiv\left(p_{z}-p_{x}\right) / \rho .
$$

The energy-momentum tensor of this fluid is then

$$
T_{\nu}^{\mu}=\operatorname{diag}(-1, w, w+3 \delta, w+3 \gamma) \rho .
$$

The Einstein equations would not allow non-diagonal components for the stresses with the metric (122).

With the matter content (123) and (125), the three nontrivial spatial components of the field equations

$$
G_{\nu}^{\mu}=8 \pi G\left(T_{(m) \nu}^{\mu}+T_{\nu}^{\mu}\right)
$$

are given as

$$
\begin{aligned}
& \frac{\ddot{b}}{b}+\frac{\ddot{c}}{c}+\frac{\dot{b} \dot{c}}{b c}=-8 \pi G\left[w_{m} \rho_{m}+w \rho\right], \\
& \frac{\ddot{a}}{a}+\frac{\ddot{c}}{c}+\frac{\dot{a} \dot{c}}{a c}=-8 \pi G\left[w_{m} \rho_{m}+(w+3 \delta) \rho\right], \\
& \frac{\ddot{a}}{a}+\frac{\ddot{b}}{b}+\frac{\dot{a} \dot{b}}{a b}=-8 \pi G\left[w_{m} \rho_{m}+(w+3 \gamma) \rho\right],
\end{aligned}
$$

and the time component is

$$
\frac{\dot{a} \dot{b}}{a b}+\frac{\dot{b} \dot{c}}{b c}+\frac{\dot{c} \dot{a}}{c a}=8 \pi G\left[\rho_{m}+\rho\right] .
$$

Overdots denote derivatives with respect to time $t$.

The continuity equations are then given by the divergence of the energy-momentum tensors. We let the two components also interact, and thus allow nonzero divergence for the individual components.

$$
\dot{\rho}_{m}+3 H\left(1+w_{m}\right) \rho_{m}=Q H \rho,
$$

and

$$
\dot{\rho}+H[3(1+w)+\delta(3-2 R+S)+\gamma(3+R-2 S)] \rho=-Q H \rho,
$$

where $Q$ describes the coupling. If there are no interactions, one finds that the densities scale as

$$
\rho_{m} \sim(a b c)^{-1-w_{m}}, \quad \rho \sim(a b c)^{-1-w} b^{3 \delta} c^{3 \gamma}
$$


The time variable we will use is an average e-folding time $x$, defined as follows:

$$
x \equiv \frac{1}{3} \log (a b c) .
$$

One notes that $H=\dot{x}$, in analogy with the FLRW case, where the e-folding time reduces to $x=\log a$. Derivative of a function $f$ with respect to $x$ will be denoted by prime, unless $f$ is explicitly specified as a function $f(y)$ of some other variable $y$.

In the following we will rewrite this system in a more convenient form for analytical and numerical study. It is then useful to follow the notation introduced in Ref. [49] by expressing the mean expansion rate as an average Hubble rate $H$

$$
H \equiv \frac{1}{3}\left(\frac{\dot{a}}{a}+\frac{\dot{b}}{b}+\frac{\dot{c}}{c}\right),
$$

and the differences of the expansion rates as the Hubble-normalized shear $R$ and $S$

$$
R \equiv \frac{1}{H}\left(\frac{\dot{a}}{a}-\frac{\dot{b}}{b}\right), \quad S \equiv \frac{1}{H}\left(\frac{\dot{a}}{a}-\frac{\dot{c}}{c}\right) .
$$

Assuming that all the expansion rates are positive, one has always $-3<R, S<3$. This is true also if either $R=0$ or $S=0$. We find then a generalized Friedmann equation of the form

$$
H^{2}=\frac{8 \pi G}{3} \frac{\rho_{m}+\rho}{1-\frac{1}{9}\left(R^{2}+S^{2}-R S\right)} .
$$

Our dynamical variables are then the density fraction $U$, and the two shear anisotropies $R$ and $S$. The evolution equations for these are then

$$
\begin{aligned}
& U^{\prime}=U(U-1)\left[\gamma(3+R-2 S)+\delta(3-2 R+S)+3\left(w-w_{m}\right)\right]-U Q,(136) \\
& S^{\prime}=\frac{1}{6}\left(9-R^{2}+R S-S^{2}\right)\left\{S\left[U\left(\delta+\gamma+w-w_{m}\right)+w_{m}-1\right]-6 \gamma U\right\},(13 \\
& R^{\prime}=\frac{1}{6}\left(9-R^{2}+R S-S^{2}\right)\left\{R\left[U\left(\delta+\gamma+w-w_{m}\right)+w_{m}-1\right]-6 \delta U\right\} .
\end{aligned}
$$

\section{Acknowledgments}

DFM acknowledges support from the A. Humboldt Foundation. We thank the anonymous referee for critical reading of the manuscript and several suggestions which have helped us to improve the paper.

\section{References}

[1] H. K. Eriksen, F. K. Hansen, A. J. Banday, K. M. Gorski, and P. B. Lilje. Asymmetries in the cmb anisotropy field. Astrophys. J., 605:14-20, 2004.

[2] G. Hinshaw et al. Three-year wilkinson microwave anisotropy probe (wmap) observations: Temperature analysis. astro-ph/0603451, 2006.

[3] Kate Land and Joao Magueijo. The axis of evil. Phys. Rev. Lett., 95:071301, 2005.

[4] Aleksandar Rakic and Dominik J. Schwarz. Correlating anomalies of the microwave sky: The good, the evil and the axis. astro-ph/0703266, 2007. 
[5] Joao Magueijo and Rafael D. Sorkin. Occam's razor meets wmap. Mon. Not. Roy. Astron. Soc. Lett., 377:L39-L43, 2007.

[6] Kate Land and Joao Magueijo. The axis of evil revisited. astro-ph/0611518, 2006.

[7] Craig J. Copi, D. Huterer, D. J. Schwarz, and G. D. Starkman. On the large-angle anomalies of the microwave sky. Mon. Not. Roy. Astron. Soc., 367:79-102, 2006.

[8] Amir Hajian and Tarun Souradeep. Testing global isotropy of three-year wilkinson microwave anisotropy probe (wmap) data: Temperature analysis. Phys. Rev., D74:123521, 2006.

[9] Christopher Gordon, Wayne Hu, Dragan Huterer, and Tom Crawford. Spontaneous isotropy breaking: A mechanism for cmb multipole alignments. Phys. Rev., D72:103002, 2005.

[10] Cristian Armendariz-Picon. Footprints of statistical anisotropies. JCAP, 0603:002, 2006.

[11] A. E. Gumrukcuoglu, Carlo R. Contaldi, and Marco Peloso. Cmb anomalies from relic anisotropy. astro-ph/0608405, 2006.

[12] Lotty Ackerman, Sean M. Carroll, and Mark B. Wise. Imprints of a primordial preferred direction on the microwave background. Phys. Rev., D75:083502, 2007.

[13] Stephon H. S. Alexander. Is cosmic parity violation responsible for the anomalies in the wmap data? hep-th/0601034, 2006.

[14] C. Armendariz-Picon. Creating statistically anisotropic and inhomogeneous perturbations. arXiv:0705.1167 [astro-ph], 2007.

[15] Christian G. Bohmer and David Fonseca Mota. Cmb anisotropies and inflation from non-standard spinors. arXiv:0710.2003 [astro-ph], 2007.

[16] Angelica de Oliveira-Costa and Max Tegmark. Cmb multipole measurements in the presence of foregrounds. Phys. Rev., D74:023005, 2006.

[17] Kaiki Taro Inoue and Joseph Silk. Local voids as the origin of large-angle cosmic microwave background anomalies. Astrophys. J., 648:23-30, 2006.

[18] Kari Enqvist and Teppo Mattsson. The effect of inhomogeneous expansion on the supernova observations. JCAP, 0702:019, 2007.

[19] Christian Armendariz-Picon. Could dark energy be vector-like? JCAP, 0407:007, 2004.

[20] C. G. Boehmer and T. Harko. Dark energy as a massive vector field. Eur. Phys. J., C50:423-429, 2007.

[21] Maxim Libanov, Valery Rubakov, Eleftherios Papantonopoulos, M. Sami, and Shinji Tsujikawa. Uv stable, lorentz-violating dark energy with transient phantom era. JCAP, 0708:010, 2007.

[22] Tomi S. Koivisto and David F. Mota. Vector field models of inflation and dark energy. 0805.4229, 2008.

[23] Tomi Koivisto and David F. Mota. Dark energy anisotropic stress and large scale structure formation. Phys. Rev., D73:083502, 2006.

[24] Richard A. Battye and Adam Moss. Anisotropic perturbations due to dark energy. Phys. Rev., D74:041301, 2006.

[25] P. R. Pereira, M. F. A. Da Silva, and R. Chan. Anisotropic self-similar cosmological model with dark energy. Int. J. Mod. Phys., D15:991-999, 2006.

[26] Kishore N. Ananda and Marco Bruni. Cosmo-dynamics and dark energy with a quadratic eos: Anisotropic models, large-scale perturbations and cosmological singularities. Phys. Rev., D74:023524, 2006.

[27] Bijan Saha. Anisotropic cosmological models with perfect fluid and dark energy revisited. Int. J. Theor. Phys., 45:952-964, 2006.

[28] Bijan Saha. Anisotropic cosmological models with perfect fluid and dark energy. Chin. J. Phys., 43:1035-1043, 2005.

[29] Valeria Pettorino, C. Baccigalupi, and F. Perrotta. Scaling solutions in scalar-tensor cosmologies. JCAP, 0512:003, 2005.

[30] Valeria Pettorino, Carlo Baccigalupi, and Gianpiero Mangano. Extended quintessence with an exponential coupling. JCAP, 0501:014, 2005.

[31] Tomi Koivisto. The matter power spectrum in $f(R)$ gravity. Phys. Rev., D73:083517, 2006.

[32] Tomi Koivisto and Hannu Kurki-Suonio. Cosmological perturbations in the palatini formulation of 
modified gravity. Class. Quant. Grav., 23:2355-2369, 2006.

[33] Timothy Clifton, David F. Mota, and John D. Barrow. Inhomogeneous gravity. Mon. Not. Roy. Astron. Soc., 358:601, 2005.

[34] Constantinos Skordis, D. F. Mota, P. G. Ferreira, and C. Boehm. Large scale structure in bekenstein's theory of relativistic mond. Phys. Rev. Lett., 96:011301, 2006.

[35] Morad Amarzguioui, O. Elgaroy, D. F. Mota, and T. Multamaki. Cosmological constraints on $f(R)$ gravity theories within the Palatini approach. Astron. Astrophys., 454:707-714, 2006.

[36] David F. Mota and Douglas J. Shaw. Strongly coupled chameleon fields: New horizons in scalar field theory. Phys. Rev. Lett., 97:151102, 2006.

[37] David F. Mota and Douglas J. Shaw. Evading equivalence principle violations, astrophysical and cosmological constraints in scalar field theories with a strong coupling to matter. Phys. Rev., D75:063501, 2007.

[38] Tomi Koivisto. Growth of perturbations in dark matter coupled with quintessence. Phys. Rev., D72:043516, 2005.

[39] Luca Amendola. Coupled quintessence. Phys. Rev., D62:043511, 2000.

[40] Robert Caldwell, Asantha Cooray, and Alessandro Melchiorri. Constraints on a new post-general relativity cosmological parameter. astro-ph/0703375, 2007.

[41] Kiyotomo Ichiki and Tomo Takahashi. Constraints on generalized dark energy from recent observations. astro-ph/0703549, 2007.

[42] D. F. Mota, J. R. Kristiansen, T. Koivisto, and N. E. Groeneboom. Constraining dark energy anisotropic stress. arXiv:0708.0830 [astro-ph], 2007.

[43] Anthony W. Brookfield, C. van de Bruck, D. F. Mota, and D. Tocchini-Valentini. Cosmology of mass-varying neutrinos driven by quintessence: Theory and observations. Phys. Rev., D73:083515, 2006.

[44] Luca Amendola, Martin Kunz, and Domenico Sapone. Measuring the dark side (with weak lensing). arXiv:0704.2421 [astro-ph], 2007.

[45] Carlo Schimd, Jean-Philippe Uzan, and Alain Riazuelo. Weak lensing in scalar-tensor theories of gravity. Phys. Rev., D71:083512, 2005.

[46] D. F. Mota and C. van de Bruck. On the spherical collapse model in dark energy cosmologies. Astron. Astrophys., 421:71-81, 2004.

[47] Nelson J. Nunes and D. F. Mota. Structure Formation in Inhomogeneous Dark Energy Models. Mon. Not. Roy. Astron. Soc., 368:751-758, 2006.

[48] Tomi Koivisto and David F. Mota. Accelerating cosmologies with an anisotropic equation of state. arXiv:0707.0279 [astro-ph], 2007.

[49] John D. Barrow. Cosmological limits on slightly skew stresses. Phys. Rev., D55:7451-7460, 1997.

[50] John D. Barrow, Yoshida Jin, and Kei-ichi Maeda. Cosmological co-evolution of yang-mills fields and perfect fluids. Phys. Rev., D72:103512, 2005.

[51] Luigi Campanelli, P. Cea, and L. Tedesco. Ellipsoidal universe can solve the cmb quadrupole problem. Phys. Rev. Lett., 97:131302, 2006.

[52] Jose Beltran Jimenez and Antonio L. Maroto. Cosmology with moving dark energy and the cmb quadrupole. astro-ph/0703483, 2007.

[53] Davi C. Rodrigues. Anisotropic cosmological constant and the cmb quadrupole anomaly. arXiv:0708.1168 [astro-ph], 2007.

[54] Michael J. Longo. Does the universe have a handedness. astro-ph/0703325, 2007.

[55] Michael J. Longo. Is the cosmic axis of evil due to a large-scale magnetic field. astro-ph/0703694, 2007.

[56] John D. Barrow and Sigbjorn Hervik. Anisotropically inflating universes. Phys. Rev., D73:023007, 2006.

[57] John D. Barrow and Sigbjorn Hervik. On the evolution of universes in quadratic theories of gravity. Phys. Rev., D74:124017, 2006.

[58] C. P. Burgess, Richard Easther, Anupam Mazumdar, David F. Mota, and Tuomas Multamaki. Multiple inflation, cosmic string networks and the string landscape. JHEP, 05:067, 2005. 
[59] Damien A. Easson, Ruth Gregory, David F. Mota, Gianmassimo Tasinato, and I. Zavala. Spinflation. JCAP, 0802:010, 2008.

[60] George F. R. Ellis and Henk van Elst. Cosmological models. gr-qc/9812046, 1998.

[61] Roman V. Buniy, Arjun Berera, and Thomas W. Kephart. Asymmetric inflation: Exact solutions. Phys. Rev., D73:063529, 2006.

[62] Bijan Saha. Interacting spinor and scalar fields in bianchi type-i universe filled with viscous fluid: Exact and numerical solutions. gr-qc/0703124, 2007.

[63] Bijan Saha. Bianchi type i universe with viscous fluid. Mod. Phys. Lett., A20:2127-2144, 2005.

[64] I. Brevik and S. D. Odintsov. On the cardy-verlinde entropy formula in viscous cosmology. Phys. Rev., D65:067302, 2002.

[65] I. Brevik and O. Gorbunova. Dark energy and viscous cosmology. Gen. Rel. Grav., 37:2039-2045, 2005.

[66] Iver Brevik. Crossing of the $\mathrm{w}=-1$ barrier in two-fluid viscous modified gravity. Gen. Rel. Grav., 38:1317-1328, 2006.

[67] Emma J. King and Peter Coles. Dynamics of a magnetised bianchi i universe with vacuum energy. Class. Quant. Grav., 24:2061-2072, 2007.

[68] Bali Raj, Pareek Umesh Kumar, and Pradhan Anirudh. Bianchi type i massive string magnetized barotropic perfect fluid cosmological model in general relativity. arXiv:0704.0753 [gr-qc], 2007.

[69] Andrew Pontzen and Anthony Challinor. Bianchi model cmb polarization and its implications for cmb anomalies. arXiv:0706.2075 [astro-ph], 2007.

[70] Shin'ichiro Ando and Marc Kamionkowski. Nonlinear evolution of anisotropic cosmological power. arXiv:0711.0779 [astro-ph], 2007.

[71] Cyril Pitrou, Thiago S. Pereira, and Jean-Philippe Uzan. Predictions from an anisotropic inflationary era. arXiv:0801.3596 [astro-ph], 2008.

[72] Camille Bonvin, Ruth Durrer, and M. Alice Gasparini. Fluctuations of the luminosity distance. Phys. Rev., D73:023523, 2006.

[73] Dominik J. Schwarz and Bastian Weinhorst. (an)isotropy of the hubble diagram: comparing hemispheres. arXiv:0706.0165 [astro-ph], 2007.

[74] Adam G. Riess et al. New hubble space telescope discoveries of type ia supernovae at $z>1$ : Narrowing constraints on the early behavior of dark energy. astro-ph/0611572, 2006.

[75] Pierre Astier et al. The supernova legacy survey: Measurement of omega $a_{m}$, omega $a_{l}$ ambda and w from the first year data set. Astron. Astrophys., 447:31-48, 2006.

[76] T. E. Perko, R. A. Matzner, and L. C. Shepley. Galaxy formation in anisotropic cosmologies. Phys. Rev., D6:969-983, 1972.

[77] H. Noh and J. C. Hwang. Perturbations of an anisotropic space-time: Formulation. Phys. Rev., D52:1970-1987, 1995.

[78] P. G. Miedema and W. A. van Leeuwen. Perturbations in bianchi i universes. Class. Quant. Grav., 9:S183-S185, 1992.

[79] E. Dimastrogiovanni, W. Fischler, and S. Paban. Perturbation Growth in Anisotropic Cosmologies. arXiv:0803.2490 [hep-th], 2008.

[80] A. E. Gumrukcuoglu, Carlo R. Contaldi, and Marco Peloso. Inflationary perturbations in anisotropic backgrounds and their imprint on the cmb. arXiv:0707.4179 [astro-ph], 2007.

[81] Thiago S. Pereira, Cyril Pitrou, and Jean-Philippe Uzan. Theory of cosmological perturbations in an anisotropic universe. arXiv:0707.0736 [astro-ph], 2007.

[82] Richard A. Battye and Adam Moss. Cosmological perturbations in elastic dark energy models. Phys. Rev., D76:023005, 2007.

[83] Peter K. S. Dunsby. Gauge invariant perturbations of anisotropic cosmological models. Phys. Rev., D48:3562-3576, 1993.

[84] Peter K. S. Dunsby. Gauge invariant perturbations in multicomponent fluid cosmologies. Class. Quant. Grav., 8:1785-1806, 1991.

[85] Christos Tsagas and Roy Maartens. Magnetized cosmological perturbations. Phys. Rev., D61:083519, 2000. 
[86] Christos G. Tsagas and Roy Maartens. Cosmological perturbations on a magnetised Bianchi I background. Class. Quant. Grav., 17:2215-2242, 2000.

[87] Christos G. Tsagas, Anthony Challinor, and Roy Maartens. Relativistic cosmology and large-scale structure. arXiv:0705.4397 [astro-ph], 2007.

[88] G. F. R. Ellis, M. Bruni, and J. Hwang. Density gradient - vorticity relation in perfect fluid robertsonwalker perturbations. Phys. Rev., D42:1035-1046, 1990.

[89] Tomi Koivisto. Viable palatini-f(r) cosmologies with generalized dark matter. Phys. Rev., D76:043527, 2007.

[90] Jose Beltran Jimenez and Antonio L. Maroto. A cosmic vector for dark energy. arXiv:0801.1486 [astro-ph], 2008. 